\title{
Variational formulation based analysis on growth of yield front in high speed rotating solid disks
}

\author{
Shubhankar Bhowmick ${ }^{1}$, Dipten Misra ${ }^{2}$ and Kashi Nath Saha ${ }^{3^{*}}$ \\ ${ }^{1}$ Faculty Member, Department of Mechanical Engineering, National Institute of Technology, Raipur 492010, India \\ ${ }^{2,3}$ Faculty members, Department of Mechanical Engineering, Jadavpur University, Kolkata 700032, India \\ *Email: kashinathsaha@gmail.com (telefax: +91 332414 6890)
}

\begin{abstract}
The present study investigates the growth of elastic-plastic front in rotating solid disks of non-uniform thickness having exponential and parabolic geometry variation. The problem is solved through an extension of a variational method in elastoplastic regime. The formulation is based on von-Mises yield criterion and linear strain hardening material behavior. Assuming a series expression of the unknown variable, the solution of the governing equation is obtained using Galerkin's principle. The approximate solution further needs an iterative method to locate the growth in the yield front. The paper reports von-Mises stress distribution in the disk at various load steps starting from the initiation of yielding till the attainment of fully plastic state. Effect of geometry parameters on the stress state of the disk is studied and the relevant results are reported in dimensionless form.
\end{abstract}

Keywords: Variational method, von-Mises stress, Yield front, Limit angular speed.

\section{Introduction}

The analysis of rotating disk behavior has been of great interest to many researchers due to its widespread engineering applications. Higher rotational speed enhances the performances of the disks and hence their demand is ever increasing in industry. Research works of rotating disks can be fundamentally classified depending on the state of stresses, i.e. elastic or elastic-plastic. It has been observed that post-elastic analysis provides a better insight towards efficient utilization of the disk material. Apart from this classification it is found that disks are generally made thick near the hub and the thickness is gradually reduced towards the periphery. The design results in variable thickness disks having higher operating speeds compared to those of constant thickness disks. Further it reduces weight and rotary inertia of the disk and hence the stress and deformation states of such disks are reported in many papers. The non-linearity associated with the study of stresses in elastic-plastic regimes is another important classification as the analysis requires advanced and efficient computational techniques, developed during the last two decades.

Theoretical analysis of rotating disks in plastic regime has long been an area of research due to its widespread applications and can be traced long back (Laszlo, 1948; Millenson and Manson, 1948; Lee 1950). For elastic and post-elastic problems the closed form solutions can also be found in standard textbooks (Timoshenko and Goodier, 1970; Calladine, 1969; Srinath, 2003 and Chakrabarty, 1987). For the post-elastic analysis of disks, although different formulations are needed for different regimes of a disk, applicability of Tresca's criterion, associated flow rule and linear strain hardening behavior presented simple and easy analytical solution and hence found mention in numerous investigations, (Gamer, 1984; Güven, 1992, 1995; Eraslan and Orcan, 2002). Gamer (1984) studied the plane stress state in a rotating disk of constant thickness of elasto-plastic material with linear strain hardening using Tresca's yield condition and its associated flow rule and reported the initiation of a plastic core at the axis of the disk. Güven (1992) extended Gamer's work to rotating annular disks with variable thickness to investigate the effect of radial density gradient on elastic-plastic stresses and radial displacement of a rotating disk with variable thickness and reported about the influence of density gradient on angular velocity and elastic plastic interface radius. Güven (1995) attempted to obtain the analytical solution for exponential variation of disk thickness assuming Tresca's criterion and its associated flow rule along with 
linear strain hardening material behavior. However a closed form solution in terms of hyper-geometric functions for exponentially varying disks was first reported by Eraslan and Orcan (2002) followed by an analytical solution for disks with parabolic thickness variation using similar functions (Eraslan 2003).

Due to non-linearity involved in use of von-Mises criterion the analysis demands for a numerical formulation yielding approximate solutions. But the advantage in using von-Mises criterion is that unlike Tresca's Criterion, here a single formulation takes care of the whole plastic region. Recently based on both Tresca's and von-Mises yield criterion, Rees (1999) presented a comparative study on the elasto-plastic behavior of rotating disks of uniform thickness made of elastic-perfectly plastic material. Based on von-Mises yield criterion, deformation theory of plasticity and a polynomial stress-strain relationship, You and Zhang (1999) presented an approximate analytical solution for rotating solid disk of uniform thickness with plane stress assumption for non-linear strain hardening material behavior. You et al. (2000) developed a unified numerical method based on polynomial stressstrain relationship, deformation theory of plasticity and von-Mises yield criterion for elasto-plastic analysis of rotating disk of varying thickness.. In another study, Eraslan (2002) studied the inelastic behavior of variable thickness rotating solid disks with linear strain hardening material behavior using both Tresca and von-Mises yield criterion. A semi analytical solution for an elasticperfectly plastic annular rotating disk and its associated flow rule was presented and the effect of yield criterion on the size of the plastic zone was reported by Alexandrova and Alexandrov (2004). In another work, Alexendrova et al. (2004) developed a semi analytical method based on von-Mises yield criterion and its associated flow rule to determine displacement field and strain distribution in a thin annular rotating disk of constant thickness. In a recent work Alexandrova and Villa Real (2007) reported the influences of rotational speed and thickness variation on the plastic solution of hyperbolic annular disks in terms of von-Mises yield criterion and its associated flow rule. Based on von-Mises failure criterion the application of variational method, proposed by Bhowmick et al. (2008) has yielded a generalized approach to study the behavior of externally loaded rotating solid disks of variable thickness in the elastic regime. In another paper (Bhowmick et al., (2009)), the method has been further extended into elasto-plastic domain.

In the present study, a numerical method based on variational principle for elasto-plastic analysis of rotating solid disks having parabolic and exponential thickness variation has been proposed. A solution algorithm using von-Mises yield criterion has been developed to obtain an approximate solution of the unknown displacement field from the governing equation in an iterative manner. The results furnish initiation of yield front and its growth with the increase in angular speed for various disk geometries.

\section{Mathematical Formulation}

In this paper, the rotational symmetry of the loading, geometry and boundary condition of the problem makes the analysis axisymmetric. Exponential and parabolic disk thickness variation is considered for the study. The rotating disk is subjected to centrifugal loading, thus producing radial and tangential strain field. At a certain speed, known as elastic limit angular speed, the stress field of the disk exceeds the yield limit value at a certain location thus giving rise to a yield front. The present method proposes a suitable iterative algorithm which can capture the location of plastic front numerically and take up the stress-strain analysis of the rotating disk. The analysis is carried out based on the assumptions that material of the disk is isotropic and homogeneous and a state of plane stress exists in the loaded condition. Stress-strain relation of the disk is considered linear elastic followed by linear strain hardening as shown in Figure 1. In the present paper, von-Mises theory is taken as the failure criteria and in case of two-dimensional stress, the general condition of yielding is given by, $\sigma_{r}^{2}-\sigma_{r} \sigma_{\theta}+\sigma_{\theta}^{2}=\sigma_{y}^{2}$. The advantage in using vonMises criterion lies in the single equation based formulation that takes care of all possible stress-state conditions in forming the plastic region.

Whenever the von-Mises stress at a particular radial location of a rotating disk reaches the uniaxial yield stress value, the plastic front initiates at that location and the corresponding rotational speed is termed as elastic limit angular speed $\left(\omega_{1}\right)$. On further increase in rotational speed, a certain region of the disk attains post-elastic state and when this region encompasses the entire disk we get plastic limit speed or collapse speed $\left(\omega_{2}\right)$. It is also important to note that the initiation of plastic front gives rise to nonhomogeneity due to presence of separate regions with different elasticity modulus values, but the assumption of isotropy remains valid for the entire disk. The von-Mises stress at elastic limit speed may be induced at root or away from the root in rotating disks depending upon the geometry of the disk. As a consequence, the growth of the post-elastic region with increase in rotational speed follows two different trends. Firstly yielding may initiate at the root of the disk and starts propagating unidirectionally towards the periphery until it attains a fully plastic state. In such a situation, the domain of the disk beyond elastic limit speed is divided into two regions (inner plastic-outer elastic). Secondly, yielding may initiate at a point away from the root and propagates bidirectionally towards root as well as the periphery. In this case the domain of the disk is divided into three regions (inner elastic intermediate plastic - outer elastic) till the plastic front reaches either at the root or the periphery. Further load increment divides the disk domain into two regions, till the disk attains fully plastic state. A mathematical formulation for the second type of disk behavior is discussed in the present text as the first case is a subset of this more generalized analysis. 


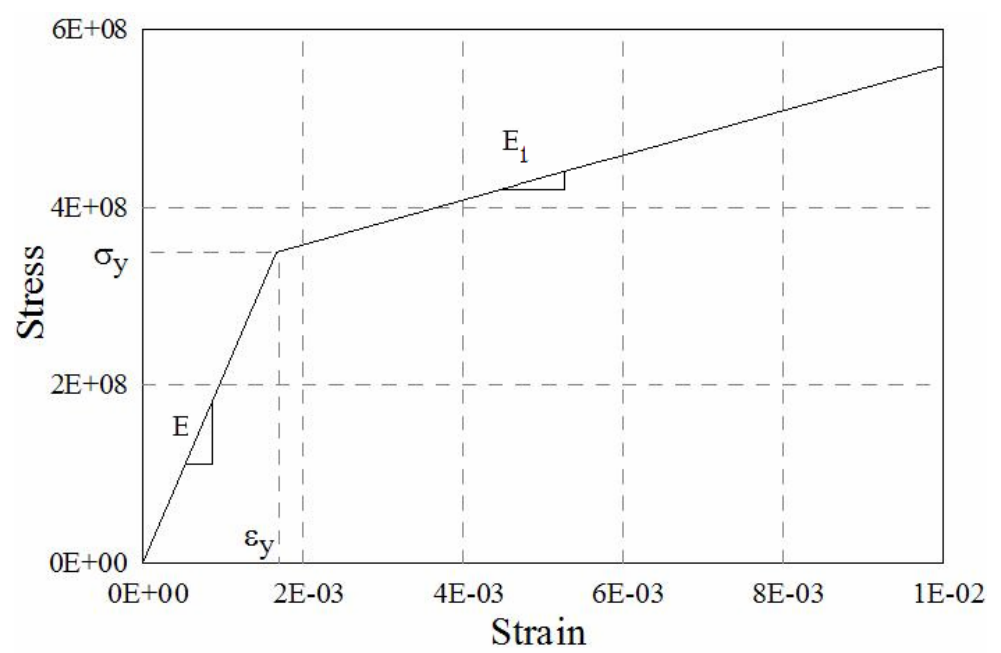

Figure 1. Linear strain hardening material behavior.

It is an established fact that (Bhowmick et al., 2008) disks with variable thickness have higher elastic limit angular speeds as compared to those of constant thickness disks and the maximum von-Mises stresses at elastic limit angular speed $\left(\omega_{1}\right)$ in such disks may occur at locations other than root. The initiation of yielding at such a location $\left(r=r_{y}\right)$ is shown in Figure 2(a) for a solid disk and the different post-elastic states of the disk with increasing angular speed are shown in Figures 2(b-e). As the centrifugal load on the disk increases, the elastic-plastic front starts to spread in two directions; towards the root as well as the periphery. At an incremented speed over the limit angular one, $\left(\omega=\omega_{1}+\Delta \omega\right)$, the plastic region spans between $r=r_{i}$ to $r=r_{o}$ as shown in Figure 2(b). Further load increment gives rise to two possibilities depending on the type of disk geometry. The plastic region may collapse at the root first (Figure 2(c)) or it may reach periphery before reaching the root (Figure 2(d)). The angular speed at which the plastic front reaches either root or periphery is designated as $\omega_{1}^{i}$ and $\omega_{1}^{o}$ respectively. Additional load increments cause the disk to attain a fully plastic state as shown in Figure 2(e) and the corresponding rotational speed is the plastic limit speed $\omega_{2}$. The form of variational principle which states that the summation of strain energy $(U)$ and work potential $(V)$ is conserved for a system in equilibrium has been used, i.e.

$$
\delta(U+V)=0
$$

Here, $U=U_{e}^{i}+U_{p}+U^{o}{ }_{e}$, i.e., total strain energy, $U$ consists of two elastic $\left(U_{e}^{i}\right.$ and $\left.U_{e}^{o}\right)$ and a plastic $\left(U_{p}\right)$ part and $V$ is the potential of the external forces. The strain energy in the disk is obtained by evaluating the integral $\frac{1}{2} \int_{V o l}(\sigma \varepsilon) d v$ and for a plane stress condition this is equivalent to computing $\frac{1}{2} \int_{V_{0}}\left(\sigma_{t} \varepsilon_{t}+\sigma_{r} \varepsilon_{r}\right) d v$. Part of strain energy in the inner elastic region (for $r=0$ to $r=r_{i}$ ) and outer elastic region (for $r=r_{o}$ to $r=b$ ) is given respectively by,

$$
\begin{aligned}
& U_{e}^{i}=\frac{\pi E}{1-v^{2}} \int_{0}^{r_{i}}\left\{\frac{u^{2}}{r}+2 v u\left(\frac{d u}{d r}\right)+r\left(\frac{d u}{d r}\right)^{2}\right\} h d r \\
& U_{e}^{o}=\frac{\pi E}{1-v^{2}} \int_{r_{o}}^{b}\left\{\frac{u^{2}}{r}+2 v u\left(\frac{d u}{d \mathrm{r}}\right)+r\left(\frac{d u}{d r}\right)^{2}\right\} h d r
\end{aligned}
$$

In arriving at these equations, the following strain-displacement and stress-strain relations have been used

$$
\begin{aligned}
\varepsilon_{r} & =d u / d r \\
\varepsilon_{t} & =u / r
\end{aligned}
$$




$$
\begin{gathered}
\sigma_{r}=\frac{E}{\left(1-v^{2}\right)}\left[\varepsilon_{r}+v \varepsilon_{t}\right] \\
\sigma_{t}=\frac{E}{\left(1-v^{2}\right)}\left[\varepsilon_{t}+v \varepsilon_{r}\right]
\end{gathered}
$$

where $u$ is the displacement field in radial direction.

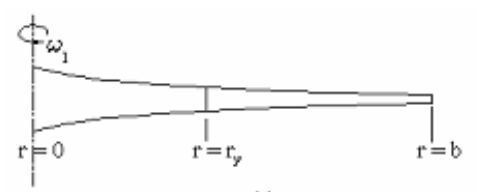

(a)

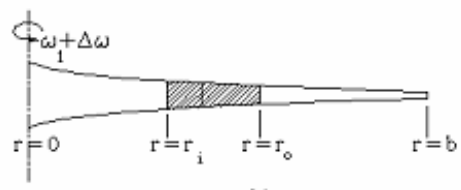

(b)

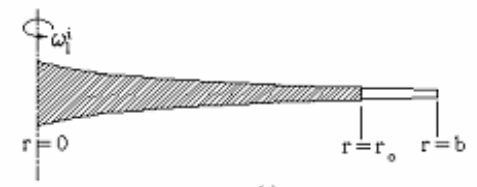

(c)

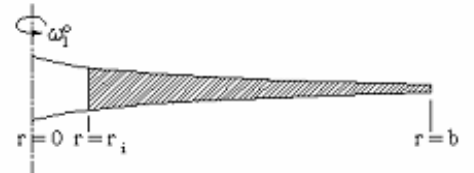

(d)

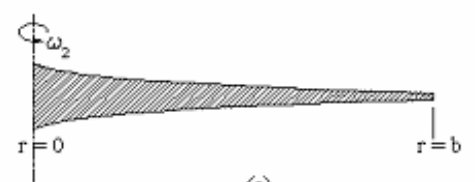

(e)

Figure 2(a-e). Propagation of elastic-plastic interface with increasing angular speed in solid disks (Post-elastic regions are indicated by hatch lines) a) at limit angular speed, b) at a speed beyond limit angular speed, c) the speed at which yield front collapses at the root, d) the speed at which yield front collapses at the periphery and e) at collapse speed when the disk is at fully plastic state

Post-elastic part of strain energy, $U_{p}$ (for $r=r_{i}$ to $r=r_{o}$ ), is obtained from the summation, i.e., $U_{p}=\int_{v o l}\left(d U_{r}+d U_{t}\right) d v$, where, $U_{r}$ and $U_{t}$ are the contributions coming from radial and tangential stresses and strains. To develop the mathematical expression of these energies, we note that the strain-displacement relations in the post-elastic region becomes,

$$
\begin{aligned}
& \frac{d u}{d r}=\varepsilon_{r}{ }^{p}+\varepsilon_{r}{ }^{0} \text { and } \\
& \frac{u}{r}=\varepsilon_{t}{ }^{p}+\varepsilon_{t}{ }^{0}
\end{aligned}
$$

Using deformation theory of plasticity (Eraslan, [12], You et al [15]) and linear strain hardening behaviour (Eraslan, 2005) the following stress-strain relations are obtained,

$$
\begin{array}{ll}
\sigma_{r}^{0}=\frac{E}{\left(1-v^{2}\right)}\left[\varepsilon_{r}^{0}+v \varepsilon_{t}^{0}\right], & \sigma_{r}^{p}=\frac{E_{1}}{\left(1-v_{p}^{2}\right)}\left[\varepsilon_{r}^{p}+v_{p} \varepsilon_{t}^{p}\right], \\
\sigma_{t}^{0}=\frac{E}{\left(1-v^{2}\right)}\left[\varepsilon_{t}^{0}+v \varepsilon_{r}^{0}\right], & \sigma_{t}^{p}=\frac{E_{1}}{\left(1-v_{p}^{2}\right)}\left[\varepsilon_{t}^{p}+v_{p} \varepsilon_{r}{ }^{p}\right] .
\end{array}
$$

Here, $\varepsilon_{r}^{0}, \varepsilon_{t}^{0}$ and $\sigma_{r}^{0}, \sigma_{t}^{0}$ are the strains and stresses in radial and tangential direction in yield condition respectively. With proper substitutions the following relations for $d U_{r}$ and $d U_{t}$ are obtained.

$$
\begin{aligned}
d U_{r}= & \frac{1}{2} \frac{E_{1}}{\left(1-v_{p}^{2}\right)}\left[\left(\frac{d u}{d r}\right)^{2}+v_{p} \frac{d u}{d r} \frac{u}{r}\right]+\frac{E}{\left(1-v^{2}\right)}\left[\varepsilon_{r}{ }^{0}+v \varepsilon_{t}^{0}\right] \frac{d u}{d r}-\frac{E_{1}}{\left(1-v_{p}{ }^{2}\right)}\left[\varepsilon_{r}{ }^{0}+v_{p} \varepsilon_{t}{ }^{0}\right] \frac{d u}{d r} \\
& +\frac{1}{2} \frac{E_{1}}{\left(1-v_{p}^{2}\right)}\left[\left(\varepsilon_{r}^{0}\right)^{2}+v_{p} \varepsilon_{r}{ }^{0} \varepsilon_{t}^{0}\right]-\frac{1}{2} \frac{E}{\left(1-v^{2}\right)}\left[\left(\varepsilon_{r}^{0}\right)^{2}+v \varepsilon_{r}{ }^{0} \varepsilon_{t}{ }^{0}\right]+\frac{1}{2} \frac{E_{1}}{\left(1-v_{p}{ }^{2}\right)} v_{p}\left[\frac{d u}{d r} \varepsilon_{t}^{0}-\frac{u}{r} \varepsilon_{r}{ }^{0}\right]
\end{aligned}
$$


and

Finally we get

$$
\begin{aligned}
d U_{t}= & \frac{1}{2} \frac{E_{1}}{\left(1-v_{p}^{2}\right)}\left[\left(\frac{u}{r}\right)^{2}+v_{p} \frac{d u}{d r} \frac{u}{r}\right]+\frac{E}{\left(1-v^{2}\right)}\left[\varepsilon_{t}^{0}+v \varepsilon_{r}^{0}\right] \frac{u}{r}-\frac{E_{1}}{\left(1-v_{p}^{2}\right)}\left[\varepsilon_{t}^{0}+v_{p} \varepsilon_{r}^{0}\right] \frac{u}{r} \\
& +\frac{1}{2} \frac{E_{1}}{\left(1-v_{p}^{2}\right)}\left[\left(\varepsilon_{t}^{0}\right)^{2}+v_{p} \varepsilon_{r}^{0} \varepsilon_{t}^{0}\right]-\frac{1}{2} \frac{E}{\left(1-v^{2}\right)}\left[\left(\varepsilon_{t}^{0}\right)^{2}+v \varepsilon_{r}^{0} \varepsilon_{t}^{0}\right]+\frac{1}{2} \frac{E_{1}}{\left(1-v_{p}^{2}\right)} v_{p}\left[\frac{u}{r} \varepsilon_{r}^{0}-\frac{d u}{d r} \varepsilon_{t}^{0}\right]
\end{aligned}
$$

$$
\begin{aligned}
& U_{p}=\left[\frac{1}{2} \frac{E_{1}}{\left(1-v_{p}^{2}\right)} \int_{r_{i}}^{r_{0}}\left\{\frac{u^{2}}{r}+r\left(\frac{d u}{d r}\right)^{2}+2 v_{p} u \frac{d u}{d r}\right\}+\frac{E}{\left(1-v^{2}\right)} \int_{r_{i}}^{r_{0}}\left\{\left(\varepsilon_{r}^{0}+v \varepsilon_{t}^{0}\right) r \frac{d u}{d r}+\left(\varepsilon_{t}^{0}+v \varepsilon_{r}^{0}\right) u\right\}\right. \\
& -\frac{E_{1}}{\left(1-v_{p}^{2}\right)} \int_{r_{i}}^{r_{0}}\left\{\left(\varepsilon_{r}^{0}+v_{p} \varepsilon_{t}^{0}\right) r \frac{d u}{d r}+\left(\varepsilon_{t}^{0}+v_{p} \varepsilon_{r}^{0}\right) u\right\}+\frac{E_{1}}{2\left(1-v_{p}^{2}\right)} \int_{r_{i}}^{r_{0}}\left\{\left(\varepsilon_{r}^{0}\right)^{2} r+\left(\varepsilon_{t}^{0}\right)^{2} r+2 v_{p} \varepsilon_{t}^{0} \varepsilon_{r}^{0} r\right\} \\
& \left.-\frac{E}{2\left(1-v^{2}\right)} \int_{r_{i}}^{r_{0}}\left\{\left(\varepsilon_{r}{ }^{0}\right)^{2} r+\left(\varepsilon_{t}^{0}\right)^{2} r+2 v \varepsilon_{t}^{0} \varepsilon_{r}{ }^{0} r\right\}\right] 2 \pi h d r
\end{aligned}
$$

Again, the work potential of the forcing function $\rho \omega^{2}$ is

$$
V=-2 \pi \rho \omega^{2} \int_{0}^{b}\left(r^{2} u h\right) d r
$$

Substituting the expressions of $U_{e}^{i}, U_{p}, U^{o}$ and $V$ in Eq.(1), the governing equilibrium equation in normalized co-ordinates becomes,

$$
\begin{aligned}
& \frac{E}{1-v^{2}} \int_{0}^{1}\left\{\frac{\Delta_{1} u \delta u}{\left(\Delta_{1} \xi_{1}+a\right)}+v\left[u \delta\left(\frac{d u}{d \xi_{1}}\right)+\frac{d u}{d \xi_{1}} \delta u\right]+\frac{\left(\Delta_{1} \xi_{1}+a\right)}{\Delta_{1}} \frac{d u}{d \xi_{1}} \delta\left(\frac{d u}{d \xi_{1}}\right)\right\} h d \xi_{1} \\
& \quad+\frac{E_{1}}{\left(1-v_{p}^{2}\right)} \int_{0}^{1}\left\{\frac{\Delta_{2} u \delta u}{\left(\Delta_{2} \xi_{2}+r_{i}\right)}+v_{p}\left[\frac{d u}{d \xi_{2}} \delta u+u \delta\left(\frac{d u}{d \xi_{2}}\right)\right]+\frac{\left(\Delta_{2} \xi_{2}+r_{i}\right)}{\Delta_{2}} \frac{d u}{d \xi_{2}} \delta\left(\frac{d u}{d \xi_{2}}\right)\right\} h d \xi_{2} \\
& \quad+\frac{E}{\left(1-v^{2}\right)} \int_{0}^{1}\left\{\varepsilon_{r}^{0}\left(v \delta u+\frac{\left(\Delta_{2} \xi_{2}+r_{i}\right)}{\Delta_{2}} \delta\left(\frac{d u}{d \xi_{2}}\right)\right)+\varepsilon_{t}^{0}\left(\delta u+v \frac{\left(\Delta_{2} \xi_{2}+r_{i}\right)}{\Delta_{2}} \delta\left(\frac{d u}{d \xi_{2}}\right)\right)\right\} h \Delta_{2} d \xi_{2} \\
& \quad-\frac{E_{1}}{\left(1-v_{p}^{2}\right)} \int_{0}^{1}\left\{\varepsilon_{r}^{0}\left(v_{p} \delta u+\frac{\left(\Delta_{2} \xi_{2}+r_{i}\right)}{\Delta_{2}} \delta\left(\frac{d u}{d \xi_{2}}\right)\right)+\varepsilon_{t}^{0}\left(\delta u+v_{p} \frac{\left(\Delta_{2} \xi_{2}+r_{i}\right)}{\Delta_{2}} \delta\left(\frac{d u}{d \xi_{2}}\right)\right)\right\} h \Delta_{2} d \xi_{2} \\
& +\frac{E}{1-v^{2}} \int_{0}^{1}\left\{\frac{\Delta_{3} u \delta u}{\left(\Delta_{3} \xi_{3}+r_{o}\right)}+v\left[u \delta\left(\frac{d u}{d \xi_{3}}\right)+\frac{d u}{d \xi_{3}} \delta u\right]+\frac{\left(\Delta_{3} \xi_{3}+r_{o}\right)}{\Delta_{3}} \frac{d u}{d \xi_{3}} \delta\left(\frac{d u}{d \xi_{3}}\right)\right\} h d \xi_{3} \\
& \quad-\rho \omega^{2} \int_{0}^{1}\left\{(\Delta \xi)^{2} h \delta u\right\} \Delta d \xi=0
\end{aligned}
$$

The normalization of Eq.(7) is carried out by using four normalized coordinates $\left(\xi, \xi_{1}, \xi_{2}\right.$ and $\left.\xi_{3}\right)$ defined as follows,

$$
\begin{aligned}
& \xi=r / b, \xi_{1}=r / r_{i}, \xi_{2}=\left(r-r_{i}\right) /\left(r_{o}-r_{i}\right), \xi_{3}=\left(r-r_{o}\right) /\left(b-r_{o}\right) \\
& \text { and } \\
& \Delta=b, \Delta_{1}=r_{i}, \Delta_{2}=r_{o}-r_{i}, \Delta_{3}=b-r_{o} .
\end{aligned}
$$

The global displacement function $u(\xi)$ in the above equation is approximated by $u(\xi) \cong \sum c_{i} \phi_{i}, i=1,2, \ldots, n_{f}$, where $\phi_{i}$ is the set of orthogonal functions developed through Gram-Schmidt orthogonalization scheme. The necessary starting function to generate the higher order orthogonal functions is selected by satisfying the relevant boundary conditions $\left(\left.u\right|_{(r=0)}=0\right.$ and $\left.\left.\sigma_{r}\right|_{(r=b)}=0\right)$ 
in elastic regime. For the purpose of computation, displacement functions in the inner elastic, post-elastic and outer elastic regions are expressed as

$$
u\left(\xi_{1}\right) \cong \sum c_{i} \phi_{i}^{e 1}, u\left(\xi_{2}\right) \cong \sum c_{i} \phi_{i}{ }^{p} \text { and } u\left(\xi_{3}\right) \cong \sum c_{i} \phi_{i}^{e 2} \text { respectively. }
$$

Substituting these assumed displacement functions and replacing operator $\delta$ by $\partial / \partial c_{j}$ the governing set of equations is obtained in matrix form as follows:

$$
\begin{aligned}
& \frac{E}{1-v^{2}} \sum_{j=1}^{n} \sum_{i=1}^{n} c_{i} \int_{0}^{1}\left\{\frac{\Delta_{1} \phi_{i}^{e l} \phi_{j}^{e l}}{\left(\Delta_{1} \xi_{1}+a\right)}+v\left(\phi_{i}^{e e^{\prime}} \phi_{j}^{e l}+\phi_{i}^{e l} \phi_{j}^{e e^{\prime}}\right)+\frac{\left(\Delta_{1} \xi_{1}+a\right)}{\Delta_{1}} \phi_{i}^{e l} \phi_{j}^{e e^{e !}}\right\} h d \xi_{1} \\
& +\frac{E_{1}}{\left(1-v_{p}^{2}\right)} \sum_{j=1}^{n} \sum_{i=1}^{n} c_{i} \int_{0}^{1}\left\{\frac{\Delta_{2} \phi_{i}^{p} \phi_{j}^{p}}{\left(\Delta_{2} \xi_{2}+r_{i}\right)}+v_{p}\left\{\phi_{i}^{p^{\prime}} \phi_{j}^{p}+\phi_{i}^{p} \phi_{j}^{p^{\prime}}\right\}+\left\{\frac{\left(\Delta_{2} \xi_{2}+r_{i}\right)}{\Delta_{2}} \phi_{i}^{p^{\prime}} \phi_{j}^{p^{\prime}}\right\}\right\} h d \xi_{2} \\
& +\frac{E}{1-v^{2}} \sum_{j=1}^{n} \sum_{i=1}^{n} c_{i} \int_{0}^{1}\left\{\frac{\Delta_{3} \phi_{i}^{e 2} \phi_{j}^{e 2}}{\left(\Delta_{3} \xi_{3}+r_{o}\right)}+v\left(\phi_{i}^{e 2^{\prime}} \phi_{j}^{e 2}+\phi_{i}^{e 2} \phi_{j}^{e 2^{\prime}}\right)+\frac{\left(\Delta_{3} \xi_{3}+r_{o}\right)}{\Delta_{3}} \phi_{i}^{e 2^{\prime}} \phi_{j}^{e e^{2}}\right\} h d \xi_{3} \\
& =\rho \omega^{2} \sum_{j=1}^{n} \int_{0}^{1}\left\{(\Delta \xi+a)^{2} \phi_{j}\right\} h \Delta d \xi-\frac{E}{\left(1-v^{2}\right)} \int_{0}^{1}\left\{\left(\varepsilon_{r}^{0} \nu \phi_{j}^{p} \Delta_{2}+\varepsilon_{r}^{0}\left(\Delta_{2} \xi_{2}+r_{i}\right) \phi_{j}^{p^{\prime}}\right)+\left(\varepsilon_{t}^{0} \phi_{j}^{p} \Delta_{2}+v\left(\Delta_{2} \xi_{2}+r_{i}\right) \varepsilon_{t}^{0} \phi_{j}^{p^{\prime}}\right)\right\} h d \xi_{2} \\
& +\frac{E_{1}}{\left(1-v_{p}^{2}\right)} \int_{0}^{1}\left\{\left(\varepsilon_{r}^{0} v_{p} \phi_{j}^{p}+\varepsilon_{r}^{0}\left(\Delta_{2} \xi_{2}+r_{i}\right) \phi_{j}^{p^{\prime}}\right) \Delta_{2}+\left(\varepsilon_{t}^{0} \phi_{j}^{p} \Delta_{2}+v_{p} \varepsilon_{t}^{0}\left(\Delta_{2} \xi_{2}+r_{i}\right) \phi_{j}^{p^{\prime}}\right)\right\} h d \xi_{2}
\end{aligned}
$$

In the above equation ()$^{\prime}$ indicates differentiation with respect to normalized coordinates.

\subsection{Solution Algorithm}

The governing equation can be expressed in matrix form as, $[K]\{c\}=\{f\}$ and the required solution of unknown coefficients, $\{c\}$ is obtained from $\{c\}=[K]^{-1}\{f\}$, solved numerically implementing an iterative scheme using IMSL subroutines. For a particular load step above elastic limit angular speed, the values of $\varepsilon_{r}{ }^{0}$ and $\varepsilon_{t}{ }^{0}$ are necessary to generate the right hand side of Eq.(10). Since the formulation is based on Hencky's total deformation theory of plasticity, the solution at each load step is carried out for the total load acting on the disk. Hence the solution at each load step involves elastic and post elastic analysis part. The ratio $(\lambda)$ of radial and tangential stress up-to yield condition is stored from the elastic part of the analysis of the converged load step. To this end, it is assumed that the ratio of radial and tangential stress when yielding occurs at present load step is same as the ratio of radial and tangential stress obtained in the preceding solved load step. This assumption is made at each point in the plastic region in the present load step. At the elastic limit angular speed if the plastic front originates at the axis then with each subsequent steps of increase in the speed, plastic front proceeds towards the outer edge. However this is valid for some specific disk profiles, whereas disks taken in the present analysis are selected such that their yielding start at an intermediate radius. Now for each load step, the location of plastic front is given a small increment starting from its previous location. From this defined limits of elastic and plastic zones the solution of the displacement field is obtained and the stress values are computed at the two assumed elasto-plastic interface. The value of von-Mises stress at the assumed plastic front location is checked for the present load step and equality of the value with that of yield stress gives the required solution for that load step. A brief description of the solution algorithm which presupposes solution of limit angular speed $\Omega_{1}$ and yield front location $r_{y}$ is provided below.

1. Three loops are commenced with $r_{i}$ and $r_{o}$ initiated to the value of $r_{y}$.

2. The rotational speed is incremented through a suitable step size $\left(\Delta \Omega_{1}\right)$ in the (first) outer loop.

3. Intermediate (second) loop is initiated and $r_{o}$ is given a small increment.

4. Inner (third) loop is initiated and $r_{i}$ is given a small decrement.

5. The ratio of $\sigma_{r}^{0}$ and $\sigma_{t}^{0}$ in the elastic region is constant for a particular radial location. That ratio ' $\lambda$ ' is stored during elastic analysis. Whenever yield front reaches a particular radial location, its von-Mises stress reaches the yield stress. Corresponding to this value of von-Mises stress, the values of ${\sigma_{r}}^{0}$ and $\sigma_{t}^{0}$ at any point in the plastic region can be obtained from the relation

$$
\sigma_{v m}=\sigma_{t}\left(1-\lambda+\lambda^{2}\right)^{\frac{1}{2}}
$$


where,

$$
\frac{\sigma_{r}}{\sigma_{t}}=\lambda
$$

6. The displacement field is then obtained using the elastic-plastic formulation of Eq. (10).

7. A check is performed on von-Mises stress and if it attains the value of yield stress at the yield front location then current position is the exact yield front location. This ensures solution for the current load step, when the relevant results are noted and followed by a load increment by returning to outer loop mentioned in step 2. Otherwise the program returns to step 3 . While noting the new locations of yield front, the possibility of its collapse at inner or outer radii is checked, and in such cases the collapse speeds $\Omega_{1}^{i}$ and $\Omega_{1}^{o}$ are obtained. When the plastic region covers whole of the disk, $\Omega_{2}$ is noted and the loops are terminated.

\subsection{Determination of Plastic Strain}

At $\Omega>\Omega_{1}$, radial and tangential strains at each coordinate inside the post-elastic region of the disk can be decomposed into elastic and plastic parts as given below.

$$
\begin{aligned}
& \varepsilon_{r}=\varepsilon_{r}{ }^{e}+\varepsilon_{r}{ }^{p} \\
& \varepsilon_{t}=\varepsilon_{t}^{e}+\varepsilon_{t}{ }^{p}
\end{aligned}
$$

where the superscript ' $e$ ' and ' $p$ ' denote the elastic and plastic part of the strains respectively.

The relations between stresses and elastic strains can be derived from generalized Hooke's law. For a state of plane stress $\left(\sigma_{z}=0\right)$, assuming Hencky's deformation theory of plasticity, the plastic strain components are given as

$$
\begin{aligned}
\varepsilon_{r}^{p} & =\frac{\mathcal{E}_{v m}^{p}}{\sigma_{v m}}\left(\sigma_{r}-\frac{\sigma_{t}}{2}\right) \\
\varepsilon_{t}^{p} & =\frac{\varepsilon_{v m}^{p}}{\sigma_{v m}}\left(\sigma_{t}-\frac{\sigma_{r}}{2}\right)
\end{aligned}
$$

In Eq. (12a, b) $\varepsilon_{v m}^{p}$ is the equivalent plastic strain and $\sigma_{v m}$ is the equivalent or von-Mises stress. Based on linear strain hardening material behavior of the disk material, the relation between equivalent plastic strain and von-Mises stress can be expressed as

$$
\varepsilon_{v m}^{p}=\left(\frac{\sigma_{v m}-\sigma_{y}}{H}\right)
$$

where $H$ is the hardening parameter and is given by

$$
H=\frac{E E_{1}}{E-E_{1}}
$$

\section{Results and Discussions}

The results are presented in terms of the following dimensionless and normalized variables: angular velocity $\Omega=\omega b \sqrt{\rho / \sigma_{y}}$, stress $\bar{\sigma}=\sigma / \sigma_{y}$, displacement $\bar{u}=u E / b \sigma_{y}$ and hardening parameter $\bar{H}=E_{1} /\left(E-E_{1}\right)$. Results for post-elastic behaviour of linear strain hardening material have been generated using the values $\bar{H}=0.5, b=1.0 \mathrm{~m}, \rho=7850 \mathrm{~kg} / \mathrm{m}^{3}, E=210 \mathrm{GPa}$ and $\sigma_{y}=350$ MPa. The value of Poisson's ratio $v$ is taken as $1 / 3$ and $v_{p}$ is taken as 0.5 .

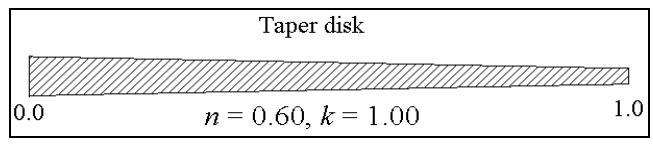

Figure 3. Tapered disk geometry used for validation 
The variation in disk geometry is controlled through parameters $n$ and $k$. The disk thickness variation is modeled mathematically using parabolic (type D1) and exponential (type D2) mathematical relations respectively as defined below:

$$
\begin{aligned}
& h(\xi)=h_{0}\left[1-n \xi^{k}\right] \text { for parabolic variation, } \\
& h(\xi)=h_{0} \exp \left[-n \xi^{k}\right] \text { for exponential disk, }
\end{aligned}
$$

For validation of the formulation a linearly tapered disk (refer Figure 3 ) is considered. The taper disk profile is obtained by considering geometry parameters $n=0.60$ and $k=1.00$ in Eq. 15. The validation of the present study is carried out with ANSYS 7.0. In ANSYS the disk of given geometry and identical material properties is modeled as an axisymmetric trapezoidal area and is meshed with plane- 82 (solid) elements with a mapped mesh density of 6 by 50 . The model is centrifugally loaded by applying global angular velocity about the axis of rotation. Firstly the propagation of yield front with increment in angular speed is validated and is plotted in dimensionless form in Figure 4. It is observed that for the selected geometry yielding initiates at a location away from the root. The normalized displacement and stress values are validated next at different load steps and are plotted in Figures 5(a-e). The meshed models along with the results of finite element analysis are shown in the appendix A, at different rotational speeds as mentioned in the next paragraph.

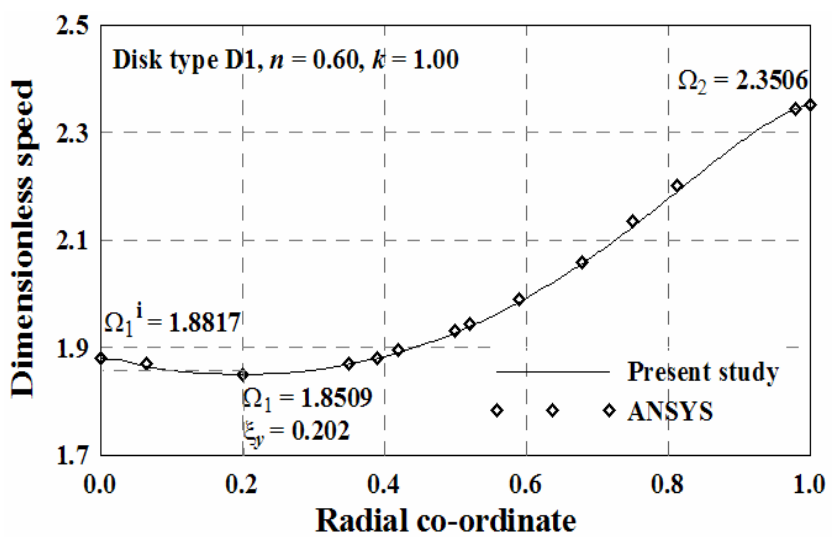

Figure 4. Propagation of yield front and its validation

The first load step selected for validation corresponds to elastic limit angular speed i.e. at $\Omega_{1}=1.8509$. It is further observed that as the plastic zone initiates at $\xi=0.20$ and starts spreading both towards root and periphery, it collapses first at the root at $\Omega_{1}{ }^{i}$ $=1.8817$. Hence it is observed that for $\Omega$ values in between 1.8509 and 1.8817 the disk domain is divided into three regions (inner elastic - intermediate plastic - outer elastic). As a result the second and the third load step selected for the validation correspond to $\Omega=1.8700$ and $\Omega=1.8817$ respectively. The fourth load step selected for validation corresponds to $\Omega=1.9291$ at which the yield front location spreading towards the periphery reaches $\xi=0.50$. The final load step for validation is selected corresponding to $\Omega_{2}$ $=2.3506$ at which the disk attains fully plastic state.

These plots exhibit very good agreement establishing validity of the present numerical scheme for the elasto-plastic analysis of high speed rotating disks. In Figure 6 the variation of normalized von-Mises stress with dimensionless angular speed for the taper disk is reported in a waterfall plot. The effect of hardening parameter $(\bar{H})$ is studied next on the disk geometry identical to the one used for validation. The comparison of yield front propagation with increasing angular speed is plotted in dimensionless form in Figure 7 for four different $\bar{H}$ values. This is followed by a comparative plot of normalized displacement and von-Mises stress at fully plastic state in Figures 8(a-b).

To carry out a parametric study of solid disk in post-elastic regime five different (constant mass) profiles each of parabolic (D1) and exponential (D2) geometries are considered. First the study is carried out for parabolic (D1) disks. The geometry parameters selected studies the effects of both $\boldsymbol{n}$ and $\boldsymbol{k}$ on the elastic and plastic limit angular speed and location of yielding. The combination of $\boldsymbol{n}$ and $\boldsymbol{k}$ values respectively considered for the study are $(0.6,0.2),(0.8,0.2),(1.0,0.2),(1.0,0.3)$ and $(1.0,0.4)$ and the profiles are shown in Figure 9. The propagation of yield front with increase in angular speed for the above mentioned geometries is plotted in Figures 10(a-e) respectively in dimensionless form, while the variation of normalized von-Mises stress with dimensionless speed for the respective geometries is shown as waterfall plots in Figures 11(a-e). 

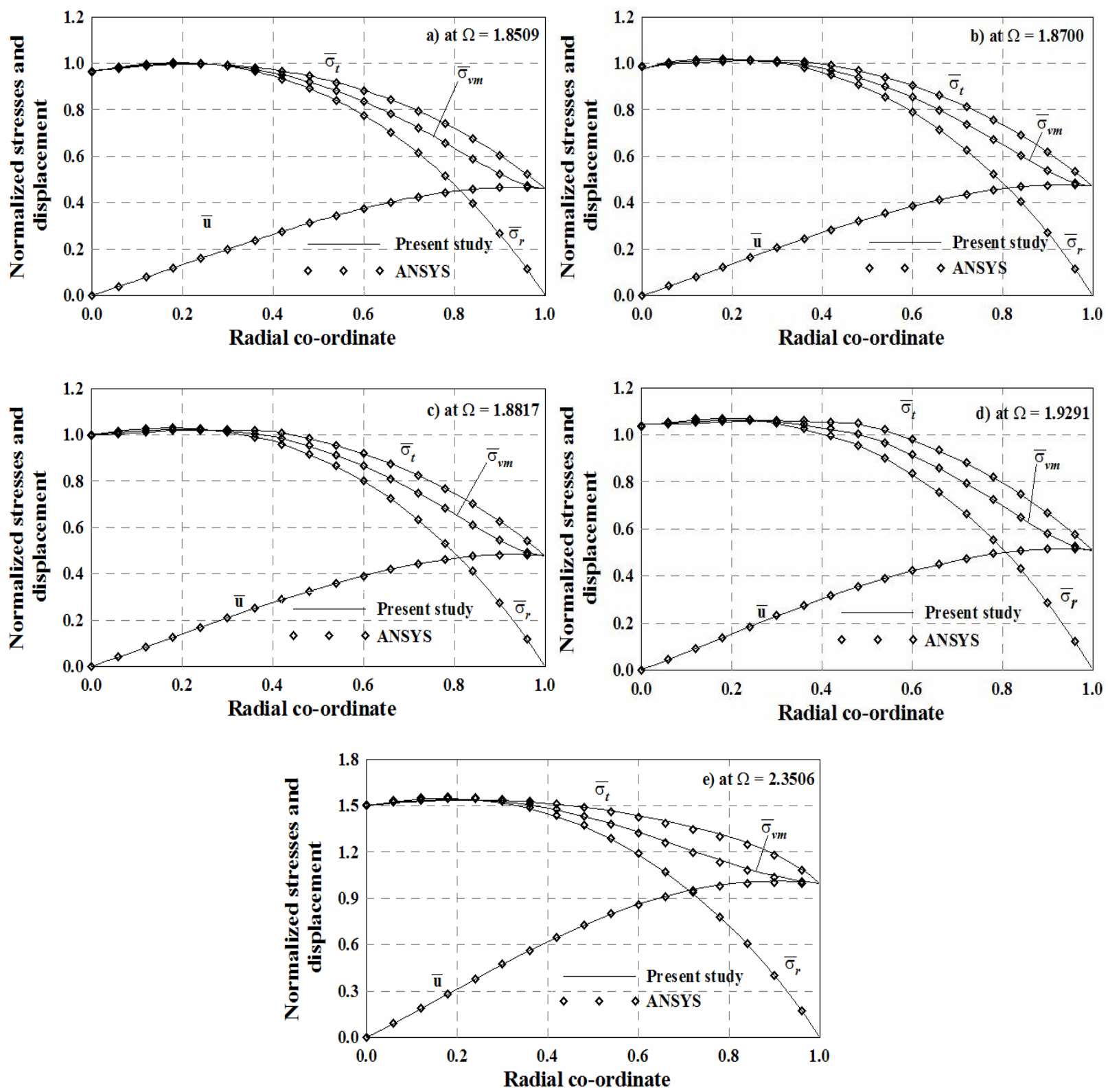

Figure 5. Normalized displacement and stress values at different angular speeds and their validation for taper disk

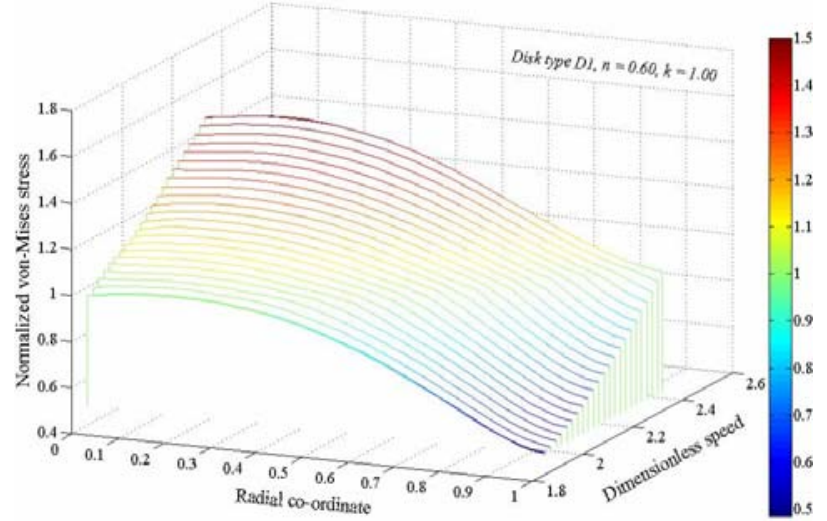

Figure 6. Waterfall plot of von-Mises stress variation with angular speed for taper disk 


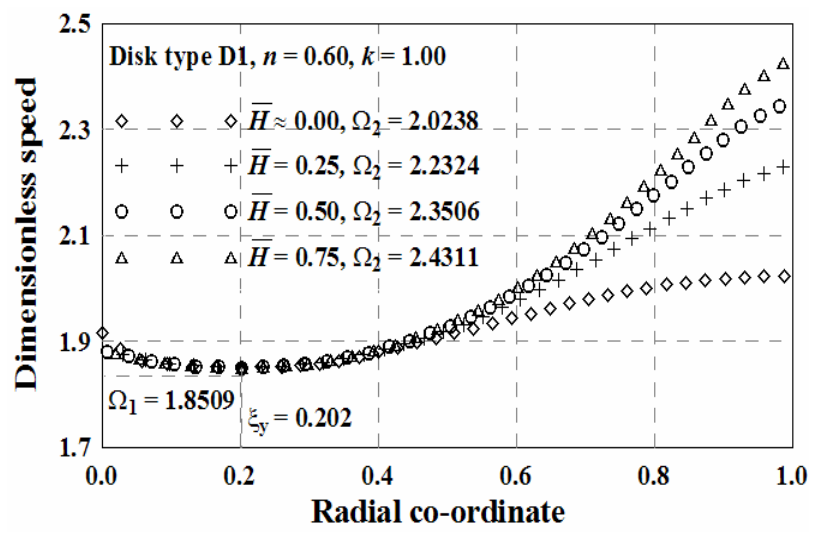

Figure 7. Comparative study of yield front propagation with angular speed for different hardening parameters
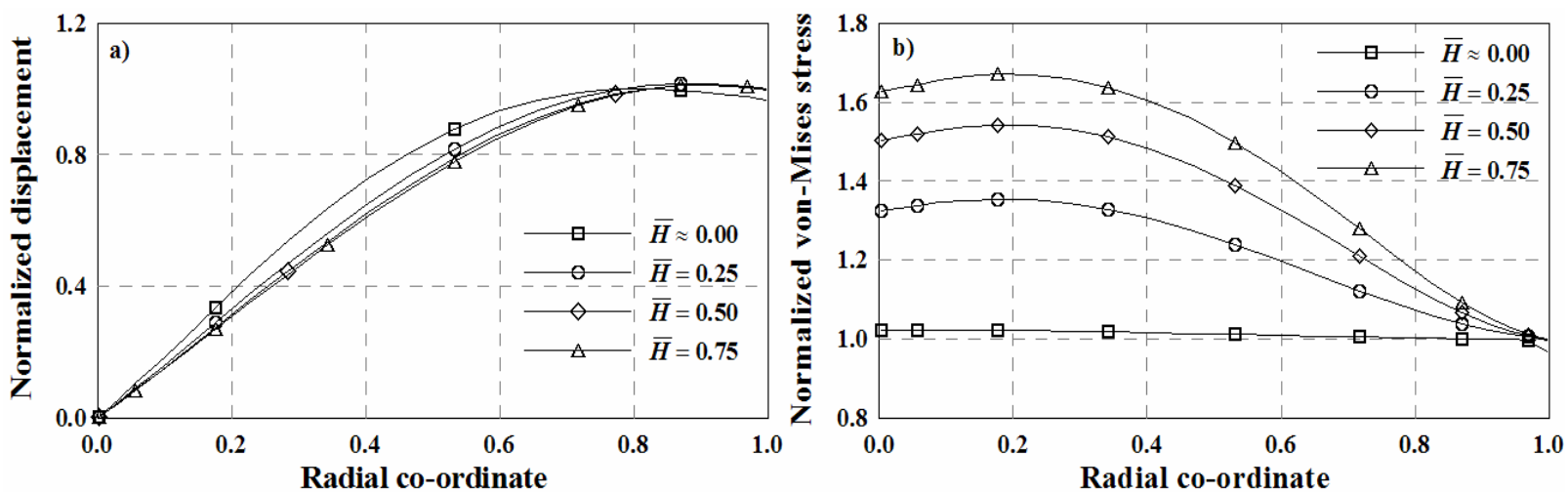

Figure 8a-b. Plots of normalized displacement and von-Mises stress for different hardening parameters
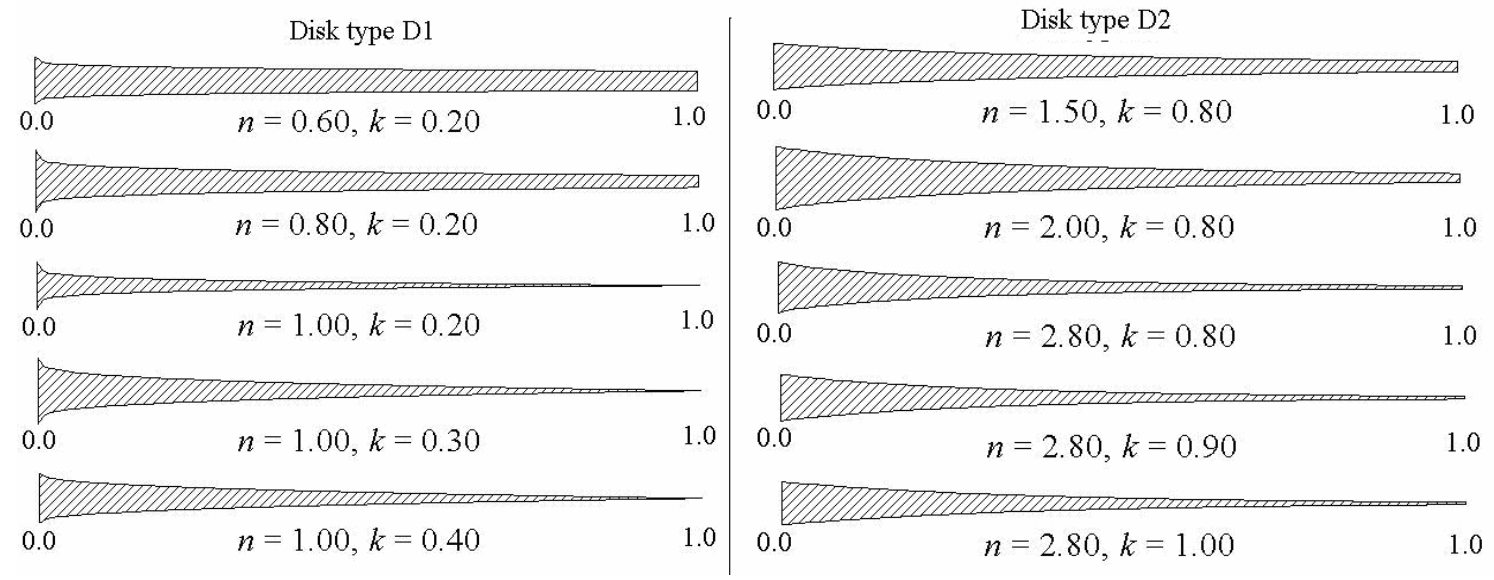

Figure 9. Various thickness profiles with corresponding geometry parameters for solid disks

In Figures 10(a-e), apart from the elastic limit speed $\left(\Omega_{1}\right)$ the partial limit plastic speed $\left(\Omega_{1}^{i}\right.$ or $\left.\Omega_{1}{ }^{o}\right)$ and fully plastic speed $\left(\Omega_{2}\right)$ are also reported. The partial limit plastic speed is the speed at which the yield front collapses at the root (at $\Omega_{1}^{i}$ ) or the periphery (at $\Omega_{1}^{o}$ ) of the disk before the disk attains fully plastic state (at $\left.\Omega_{2}\right)$. It is observed from Figure 10(a, b and c) that for a given value of parameter $\boldsymbol{k}$, with increase in $\boldsymbol{n}$ the location of yield initiation shifts towards the periphery. This results in higher fully plastic speeds as indicated in the figures thus resulting into higher strength disks. In Figures $10(\mathrm{c}, \mathrm{d}$ and e) it is observed that for a given $\boldsymbol{n}$, increase in $\boldsymbol{k}$ shifts the location of yield initiation towards the root of the disk. This results in reduced fully plastic speed. 
The normalized radial and tangential plastic strains at corresponding partial limit plastic speed are plotted in Figures 12(a-b) where as identical plots of normalized radial and tangential plastic strains at fully plastic speed for the same disks are plotted in Figures 13(a-b). The values of the respective partial limit speed and fully plastic speed for the respective geometries is indicated in the legends of the figure.

Next a similar study is carried out for exponential (D2) disks. The corresponding set of $\boldsymbol{n}$ and $\boldsymbol{k}$ values defining the disk geometry are $(1.5,0.8),(2.0,0.8),(2.8,0.8),(2.8,0.9)$ and $(2.8,1.0)$. The propagation of yield front with increase in angular speed in dimensionless form for each set is shown in Figures 14(a-e) respectively. The effect of increase in parameter $\boldsymbol{n}$ for a given value of $\boldsymbol{k}$ on the behavior of disk type D2 is observed from Figure 14(a,b and c) to be similar to that of type D1. With increase in $\boldsymbol{n}$ the location of yield initiation shifts towards the periphery, thus increasing the fully plastic speed. However the effect of variation in $\boldsymbol{k}$ for a given value of $\boldsymbol{n}$ on the disk behaviour for disk type D2 is different than that of D1. It is observed from Figure 14(c, d and e) that, with increase in $\boldsymbol{k}$ for a given value of $\boldsymbol{n}$ the location of yield initiation of disk type D2 shifts towards the periphery of the disk. An explanation of this behavior can be obtained from the understanding of variation in mass distribution resulting from the variation of $\boldsymbol{n}$ and $\boldsymbol{k}$ for disk type D2 (Eq. 16). The waterfall plots of normalized von-Mises stress with dimensionless speed for each set is shown in Figures 15(a-e) respectively. Normalized radial and tangential plastic strains at partial limit plastic speed and fully plastic speed are plotted in Figures 16(a-b) and Figures 17(a-b) respectively for disk type D2.
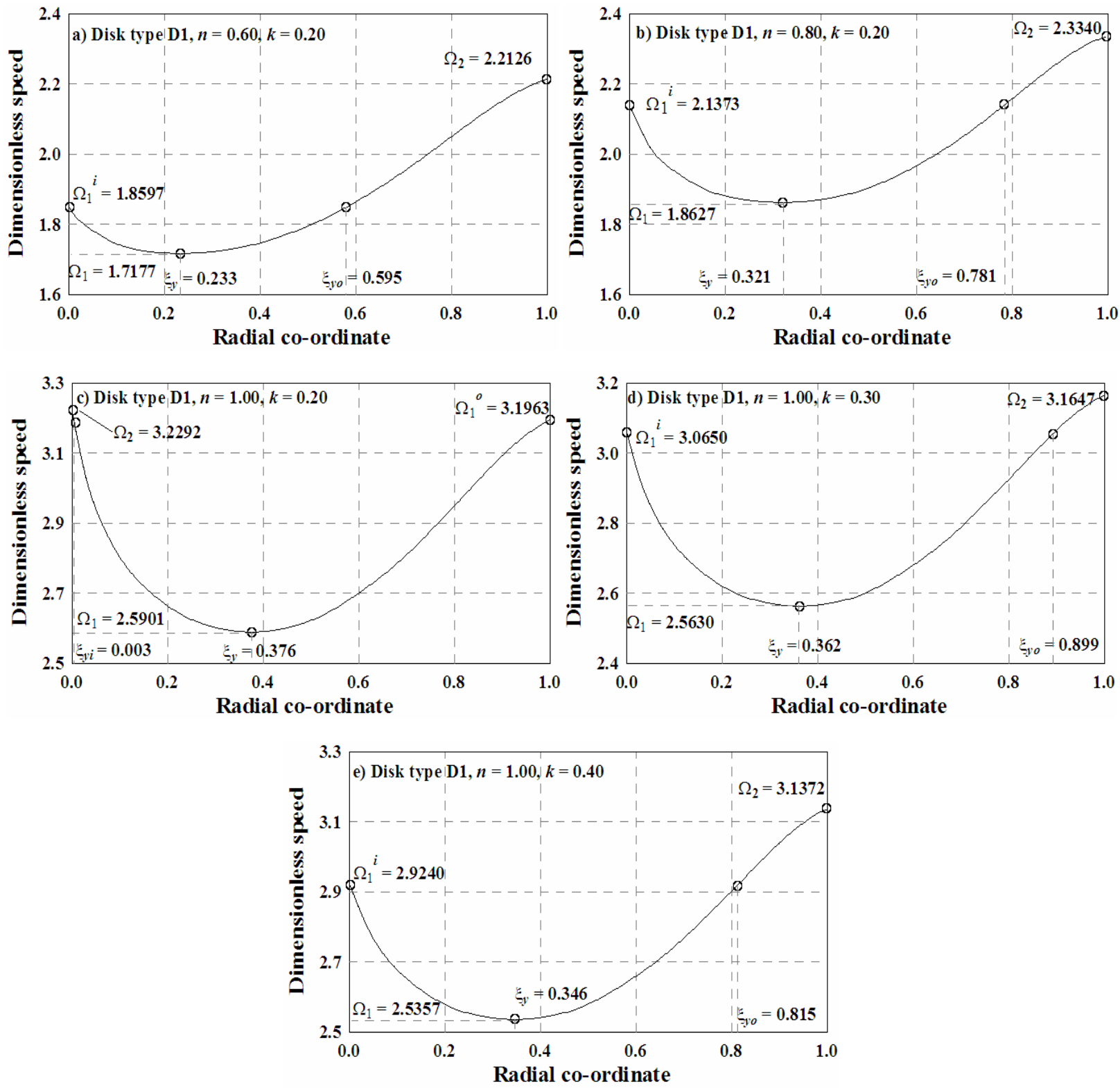

Figure 10a-e. Plot of propagation of yield front with increase in angular speed for disks of type D1 


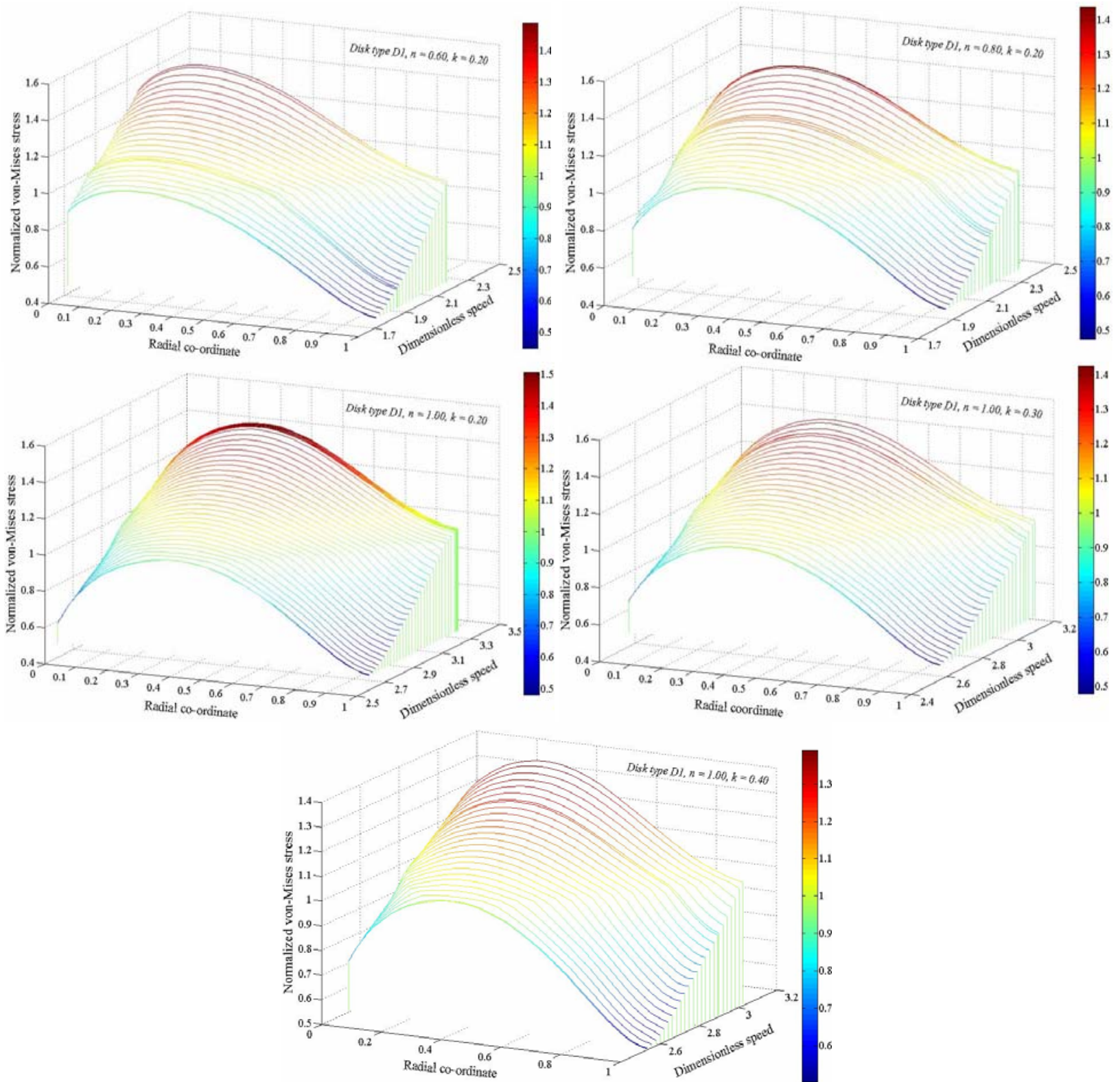

Figure 11a-e. Waterfall plots of von-Mises stress variation with angular speed for disks of type D1
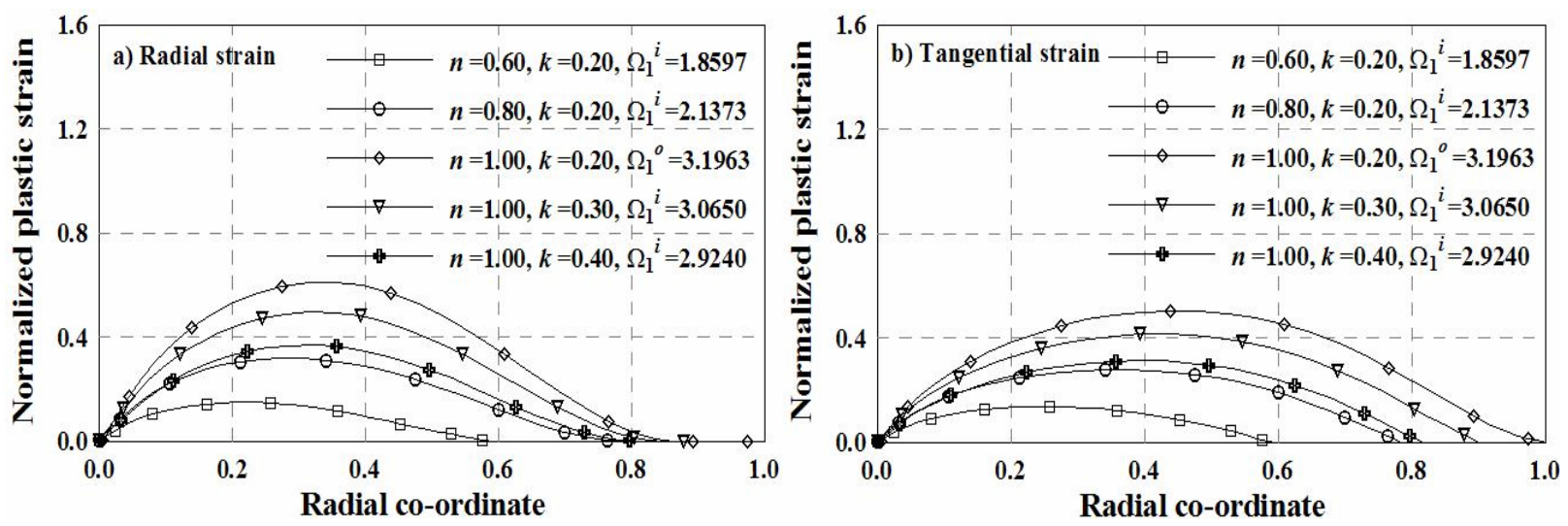

Figure 12a-b. Normalized radial and tangential plastic strain for disks of type D1 at partial limit plastic speeds 

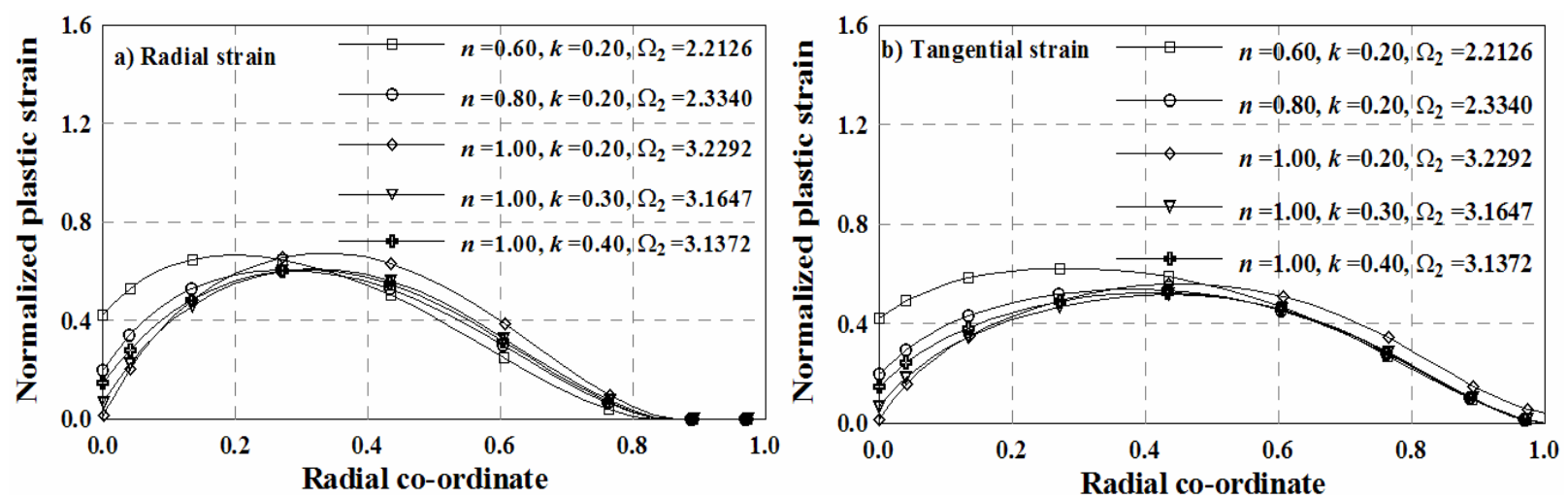

Figure 13a-b. Normalized radial and tangential plastic strain for disks of type D1 at fully plastic speeds
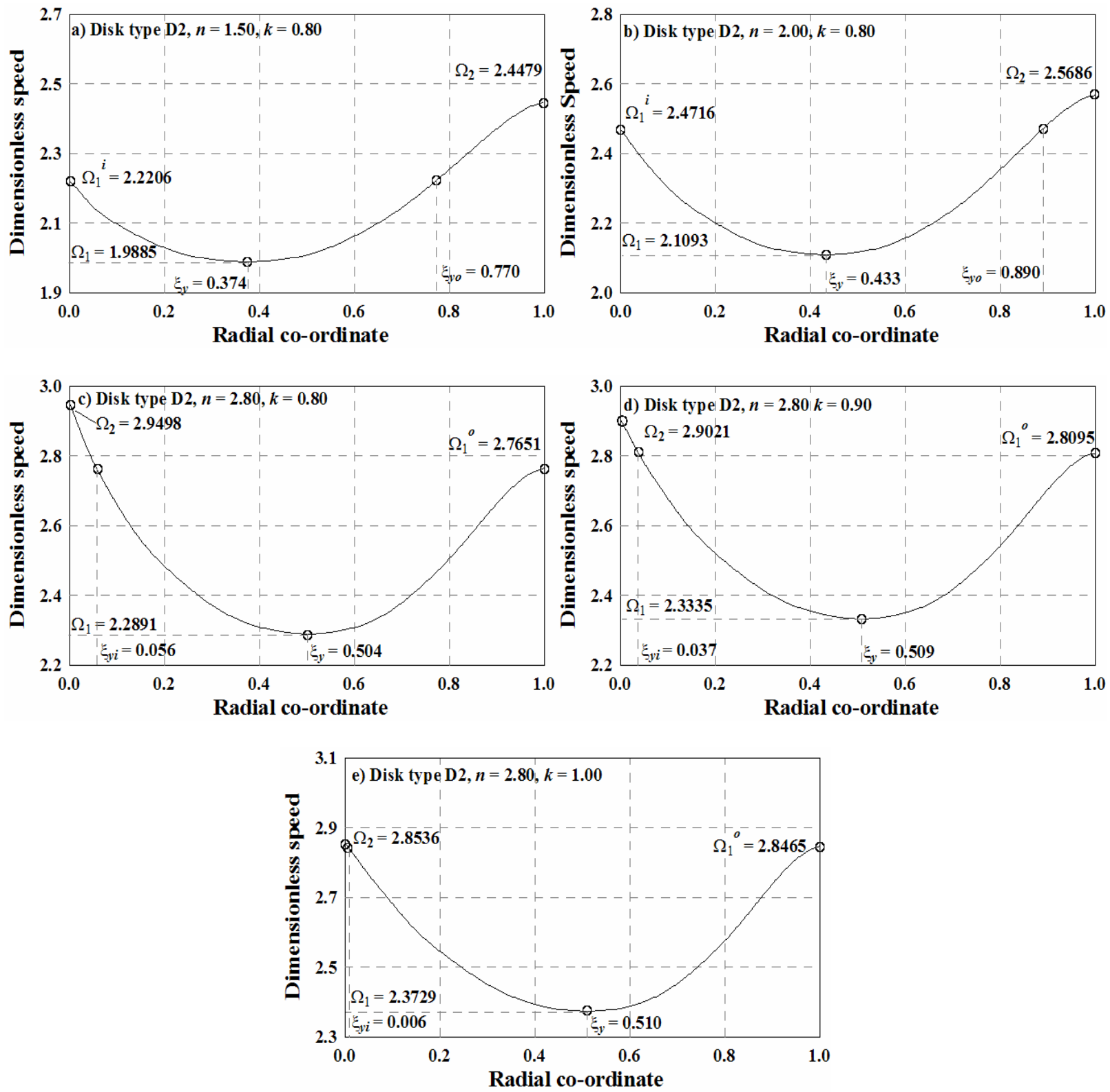

Figure 14a-e. Plot of propagation of yield front with increase in angular speed for disks of type D2 

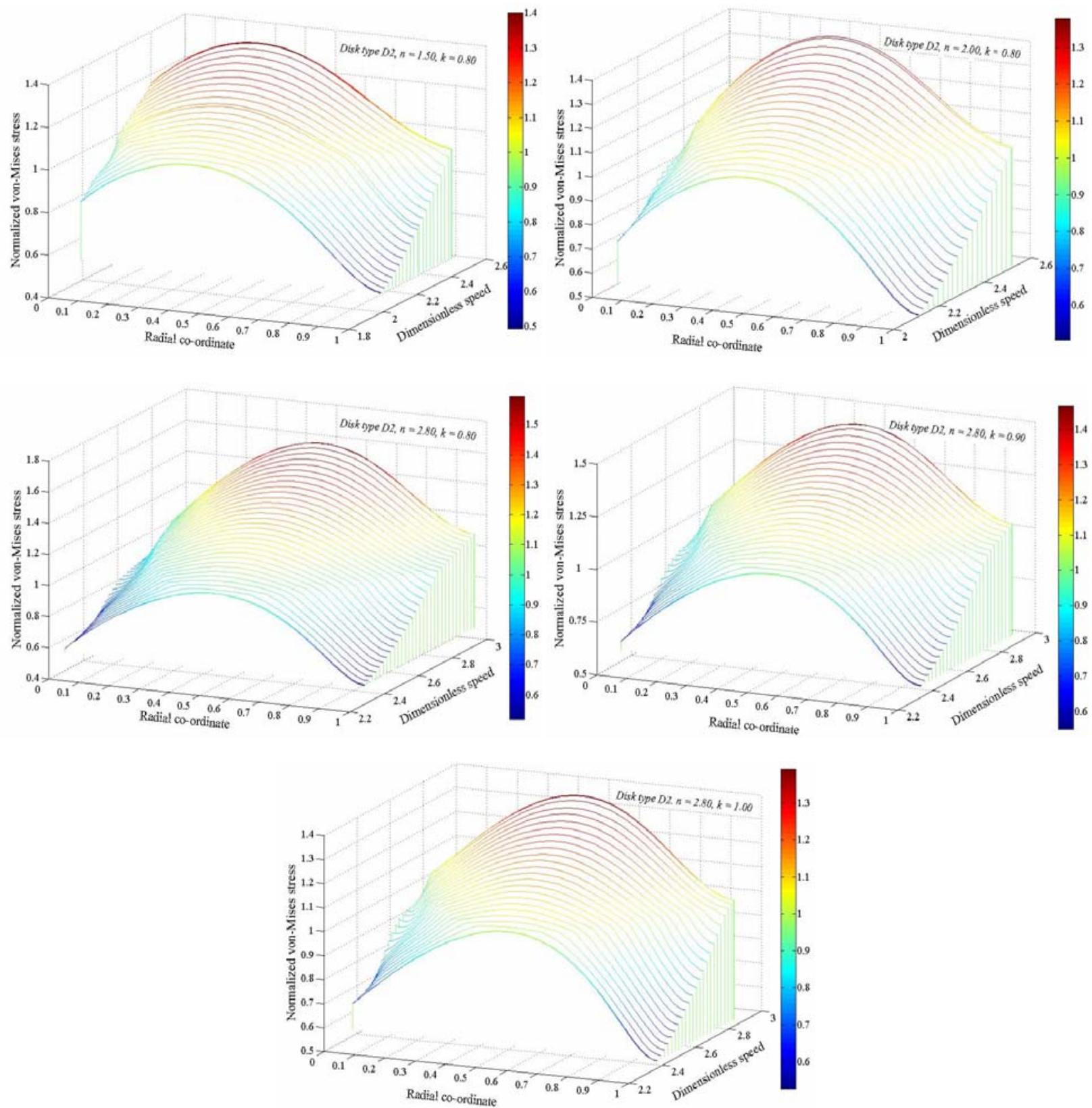

Figure 15a-e. Waterfall plots of von-Mises stress variation with angular speed for disks of type D2
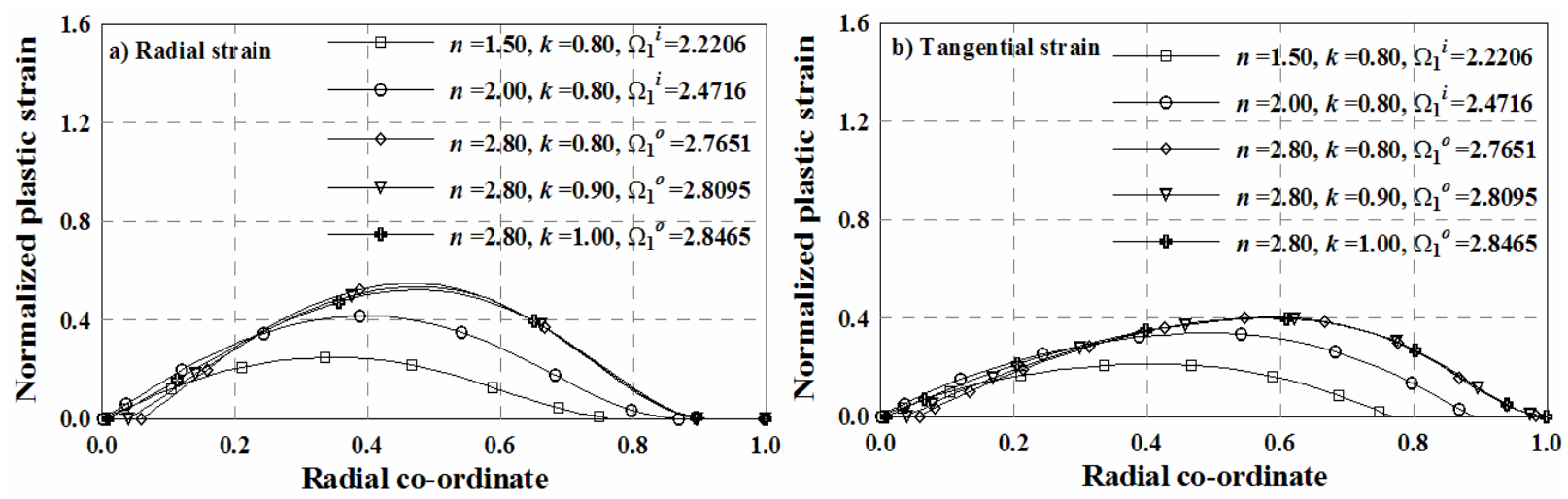

Figure 16a-b. Normalized radial and tangential plastic strain for disks of type D2 at partial limit plastic speeds 
The operating range of rotating disk is defined by its fully plastic speed. It is observed from the Figures 10-14 (a-e) that for disk types D1 and D2, for a given value of parameter $\boldsymbol{k}$, increase in $\boldsymbol{n}$ results in increase of partial limit and fully plastic speed thus increasing the operating range. However for both disk types, it is observed (Figures 10c-e and Figures 14c-e) that for a given value of parameter $\boldsymbol{n}$, increase in $\boldsymbol{k}$ results in lower fully plastic speeds thus reducing the operating range of the disk in post-elastic state. In general, the difference of fully plastic and limit elastic speed can be used as an index to measure the extent of effective utilization of disk material due to its thickness variation. On this basis it is observed that among the selected disk profiles the most effective ones are of geometry parameters $\boldsymbol{n}=1.0$ and $\boldsymbol{k}=0.2$ for disk type D1 and of $\boldsymbol{n}=2.8$ and $\boldsymbol{k}=0.8$ for disk type D2.

The response of limit elastic and fully plastic speed to variation in geometry parameter is observed to be similar for disk types D1 and D2. The variation in partial limit plastic speed and fully plastic speed with geometry parameters understandably affects the magnitude of plastic strain components for each geometry as is observed in Figures 12(a-b) and 13(a-b) for disk type D1 and Figures 16(a-b) and 17(a-b) for disk type D2 respectively.
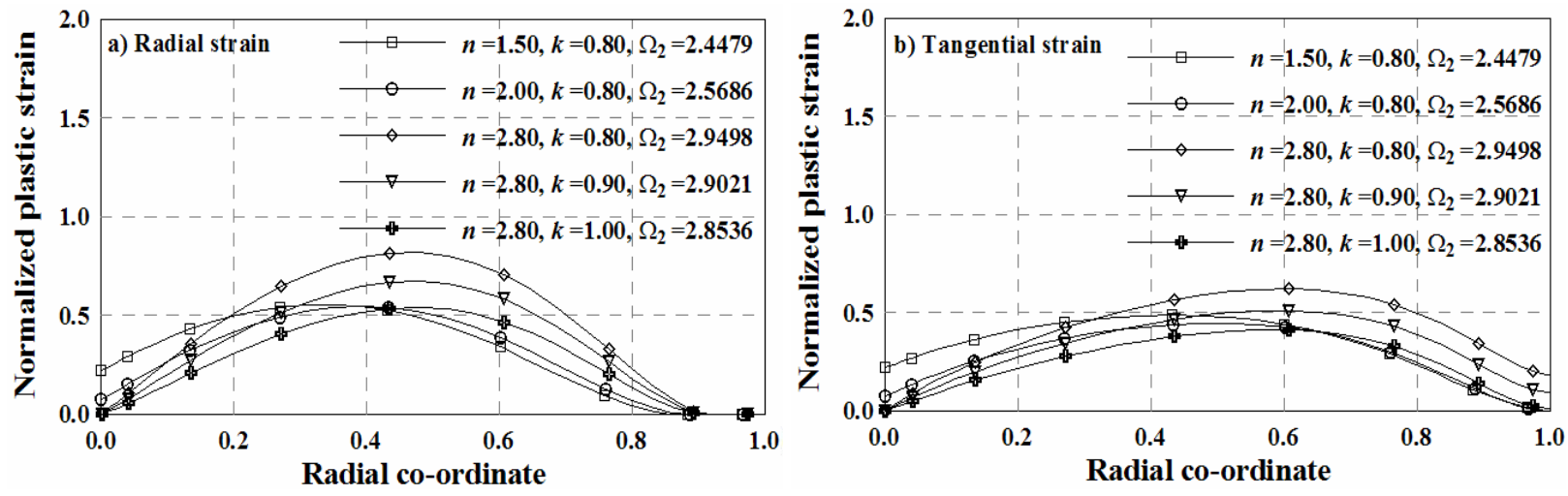

Figure 17a-b. Normalized radial and tangential plastic strain for disks of type D2 at fully limit plastic speeds

\section{Conclusion}

The investigation of yield front propagation of variable geometry rotating disks is formulated through a variational method assuming linear strain hardening material behavior following von-Mises yield criterion. The results obtained by the present methodology have been validated and it showed a close conformity with the existing results of similar problem. Some new results for yield front propagation for different geometries of rotating disks have been furnished and waterfall plots showing the vonMises stress field with increase in rotational speed have been presented. The results are presented graphically so that they become designer friendly. The method developed has application potential in various other problems, e.g., shrink fitted rotating disk, prestressed rotating disk, compound disk made from different materials, etc. The method of formulation also gives the kernel for dynamic analysis and in the analysis of many other complicating effects.

\section{Acknowledgement}

The present study is financially supported by CSIR, India (Ref. File/letter No. 9/96[514]2K7-EMR-I) and the support is duly acknowledged by the first author.

\section{Nomenclature}

b Outer radius of the disk

$\mathrm{c}_{\mathrm{i}} \quad$ The vector of unknown coefficients

E Elasticity modulus of the disk material

$\mathrm{E}_{1} \quad$ Tangent modulus of the disk material

\{f $\quad$ Load vector

$\mathrm{h}_{0} \quad$ Thickness at the root of the disk

$\mathrm{h} \quad$ Thickness at any radius $\mathrm{r}$ of the disk

$\bar{H} \quad$ Normalized hardening parameter

[K] Stiffness matrix

$\mathrm{n}, \mathrm{k} \quad$ Geometry parameters

$\mathrm{n}_{\mathrm{f}} \quad$ Number of co-ordinate functions

$r_{y} \quad$ Location of yield front at yield initiation

$r_{i} \quad$ Location of yield front spreading towards root 
$r_{o} \quad$ Location of yield front spreading towards periphery

$\mathrm{u} \quad$ Displacement field of the disk

U Strain energy of the disk

$\mathrm{U}_{\mathrm{p}} \quad$ Strain energy in the plastic part of the disk

$\mathrm{U}_{\mathrm{e}}{ }_{\mathrm{i}}, \mathrm{U}_{\mathrm{e}}{ }^{0}$ Strain energy in the inner and outer elastic part of the disk

$\mathrm{V} \quad$ Work potential of the disk because of rotation

$\beta \quad$ Ratio of tip thickness to root thickness

$\delta \quad$ Variational operator

$\rho \quad$ Density of the disk material

$v \quad$ Poisson's ratio of the disk in elastic state

$v_{\mathrm{p}} \quad$ Poisson's ratio of disk in post-elastic state

$\omega \quad$ Dimensional angular speed of the disk

$\Omega \quad$ Normalized angular speed of the disk

$\Omega_{1} \quad$ Normalized limit elastic angular speed

$\Omega_{2} \quad$ Normalized fully plastic angular speed

$\Omega_{1}{ }^{i} \quad$ Normalized angular speed corresponding to collapse of yield front at root

$\Omega_{1}{ }^{0} \quad$ Normalized angular speed corresponding to collapse of yield front at periphery

$\varepsilon_{r}, \varepsilon_{t} \quad$ Radial and tangential strains respectively

$\varepsilon_{r}^{0}, \varepsilon_{t}^{0} \quad$ Radial and tangential strains in yield condition

$\varepsilon_{r}^{p}, \varepsilon_{t}^{p}$ Radial and tangential strains above yield strain

$\varepsilon_{v m}^{p} \quad$ Equivalent plastic strain

$\sigma_{r}, \sigma_{t} \quad$ Radial and tangential stresses

$\sigma_{r}^{0}, \sigma_{t}^{0}$ Radial and tangential stress in yield condition

$\sigma_{r}^{p}, \sigma_{t}^{p}$ Radial and tangential stress above yield stress

$\sigma_{v m} \quad$ von-Mises stress

$\sigma_{y} \quad$ Yield stress of the disk material

$\phi_{i} \quad$ Set of orthogonal functions

$\xi \quad$ Normalized global radial co-ordinate

$\lambda \quad$ Ratio of $\sigma_{r}$ and $\sigma_{t}$

\section{Appendix A:}

The meshed model and the finite element validation results as carried out in Ansys

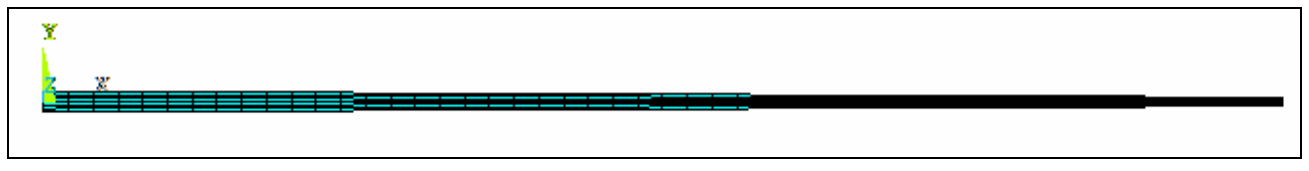

Figure 18. Meshed geometry of taper disk obtained in Ansys

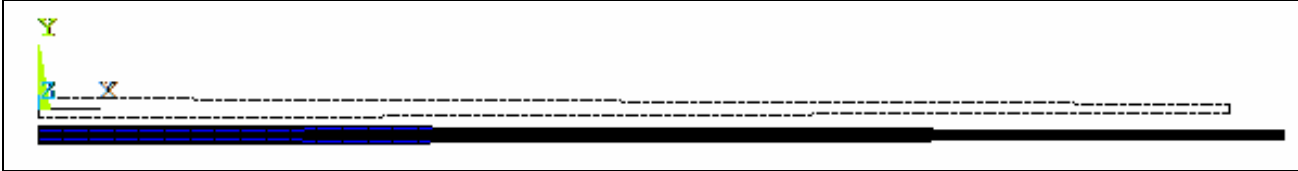

Figure 19. Undeformed edge and deformed geometry at a elastic limit speed (at $\Omega_{1}=1.8509$ ) 


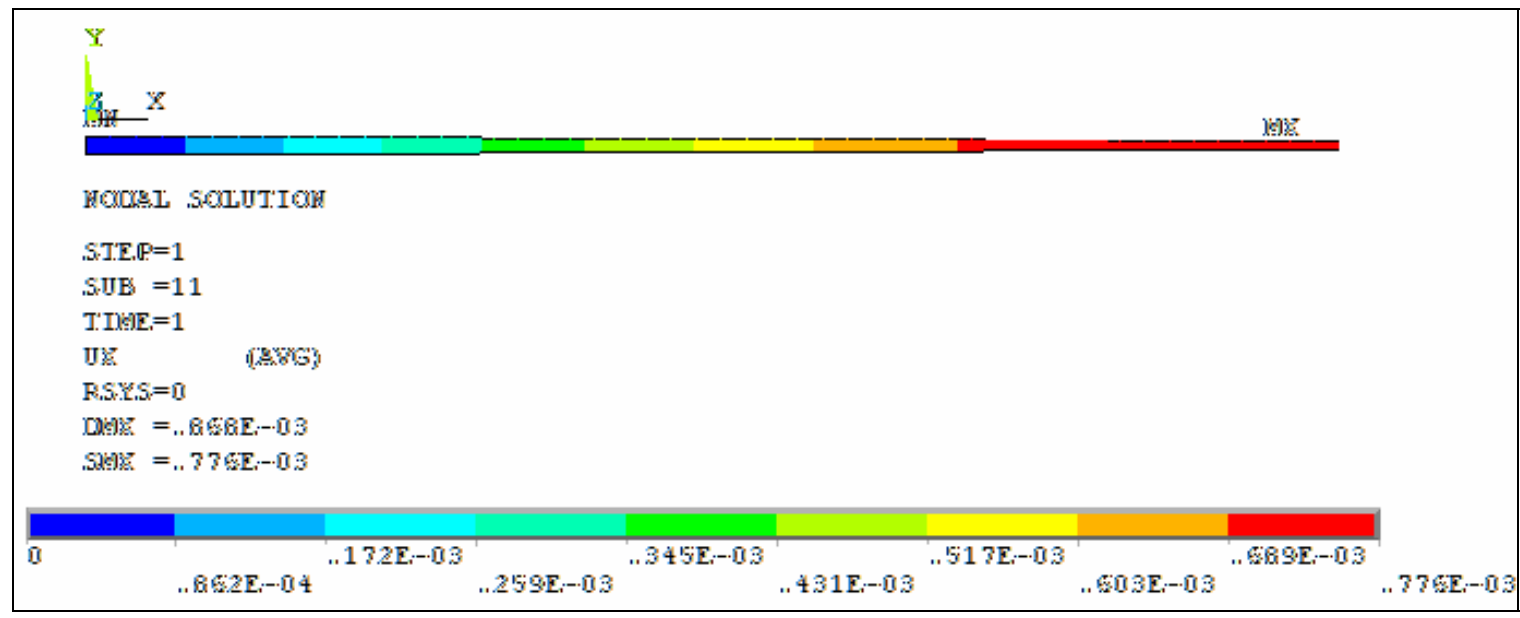

a) Plot of radial displacement at the elastic limit speed (at $\Omega_{1}=1.8509$ )

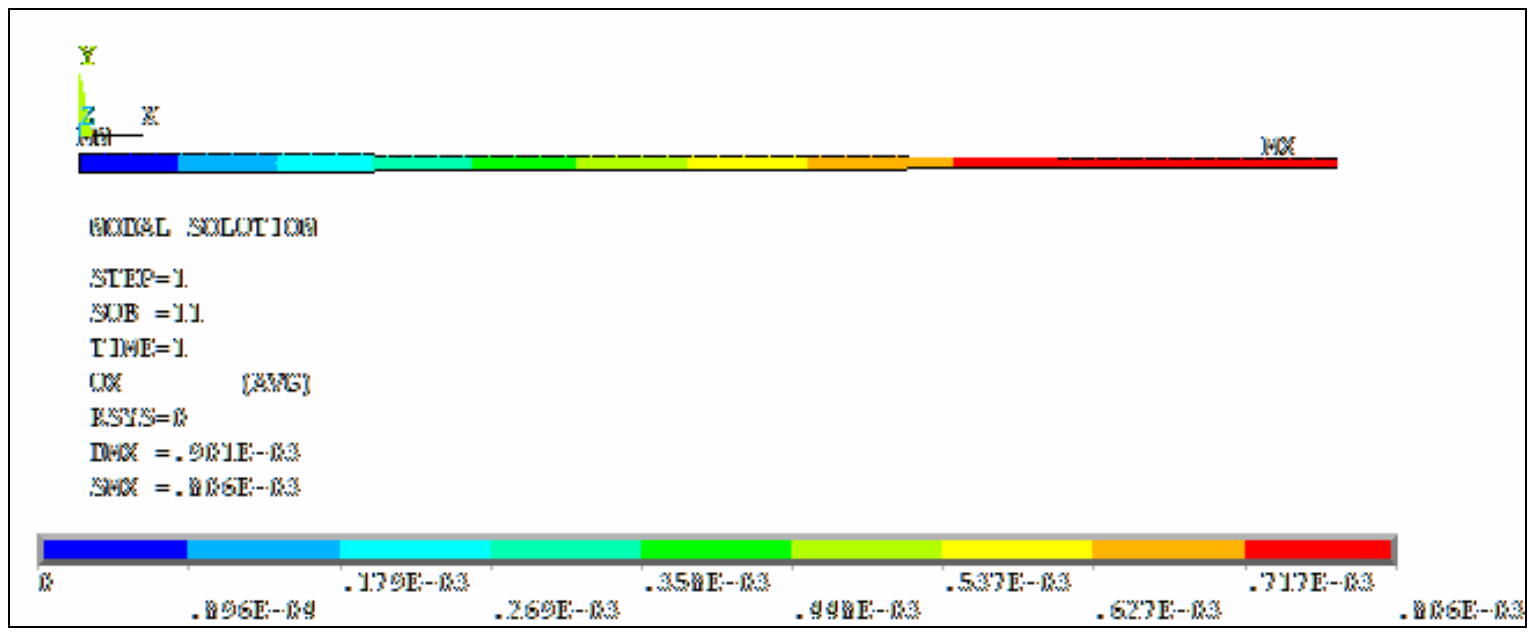

b) Plot of radial displacement at post limit speed (at $\Omega=1.8817$ )

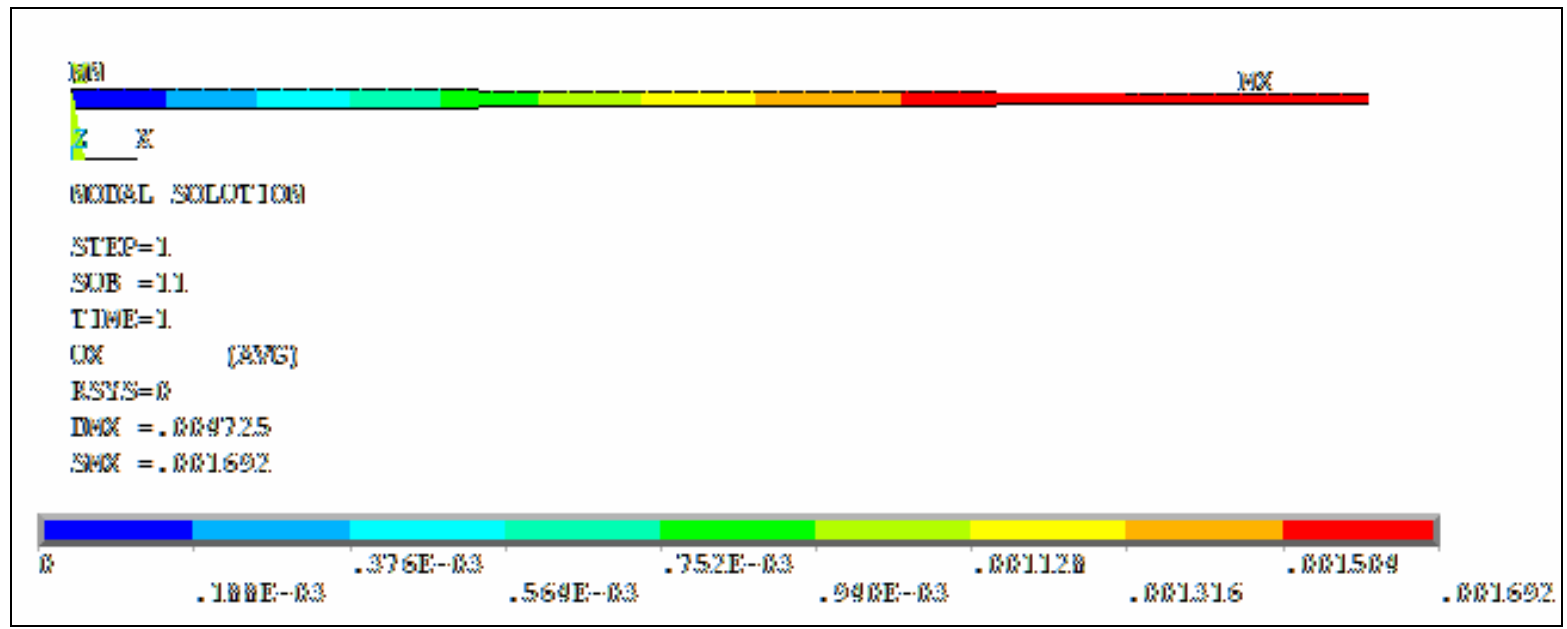

c) Plot of radial displacement at fully plastic speed (at $\Omega_{2}=2.3506$ )

Figure 20. The finite element analysis of radial displacement fields at different rotational speeds. 


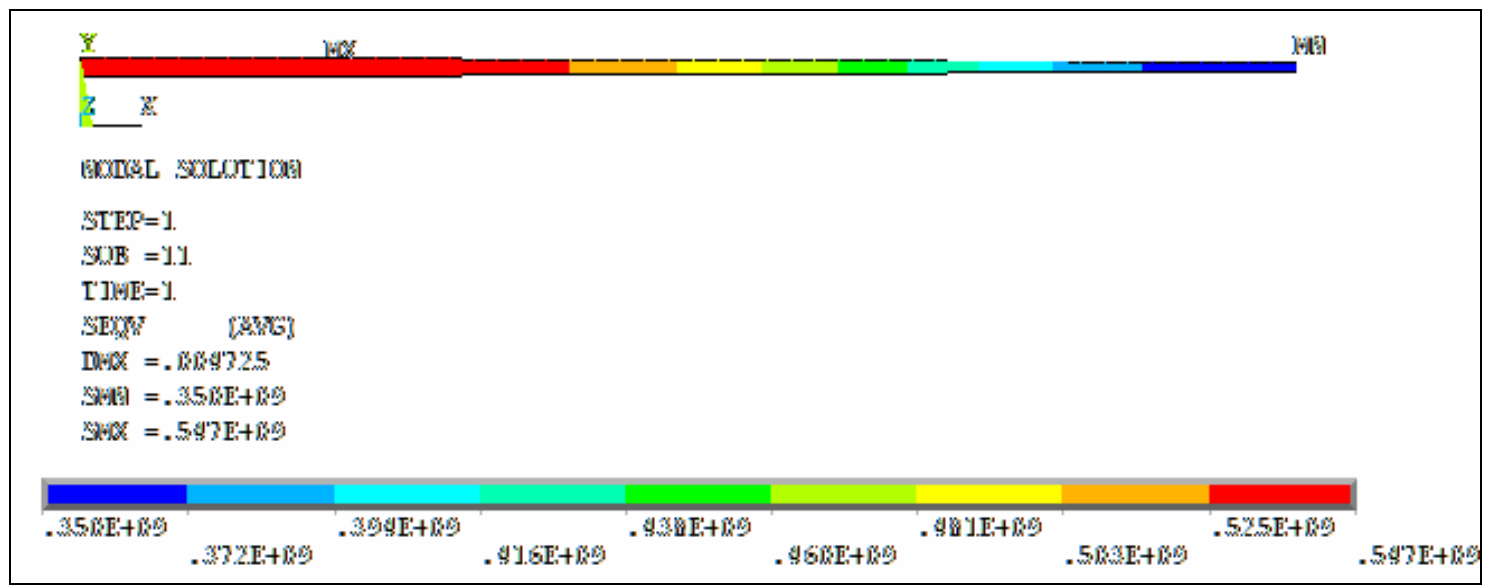

a) Plot of von-Mises stress at the elastic limit speed (at $\Omega_{1}=1.8509$ )

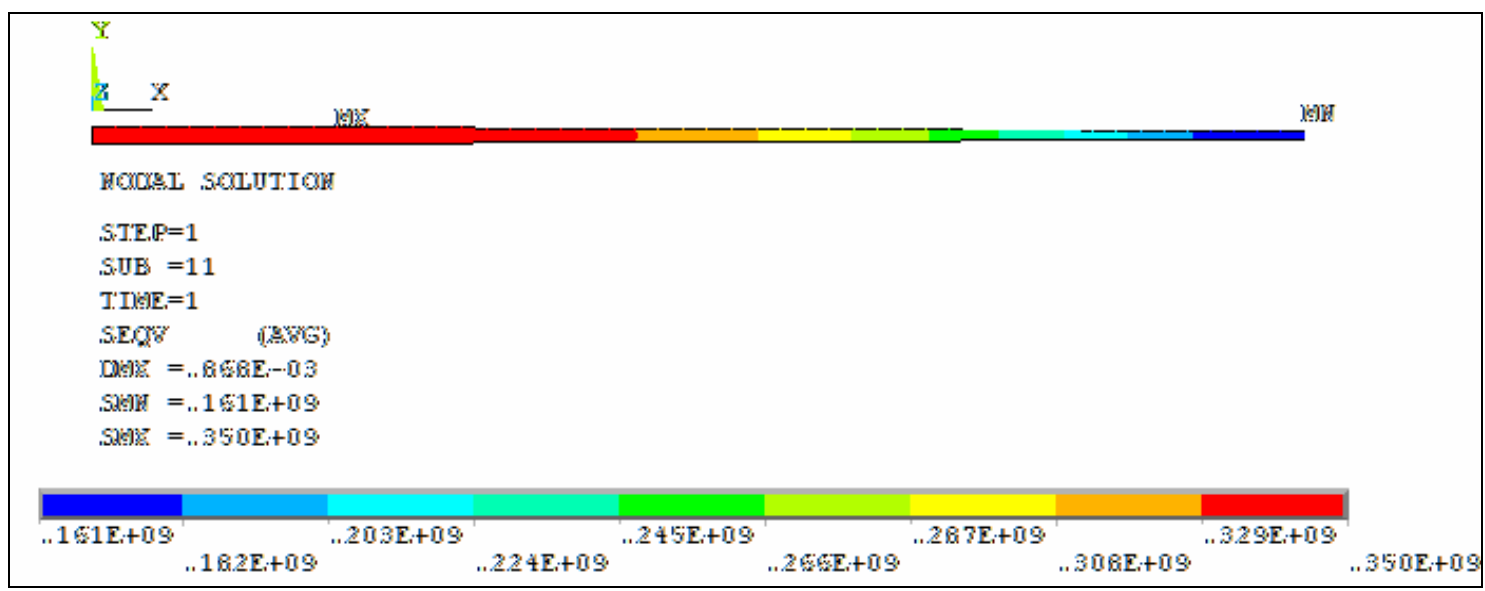

b) Plot of von-Mises stress at post limit speed (at $\Omega=1.8817$ )

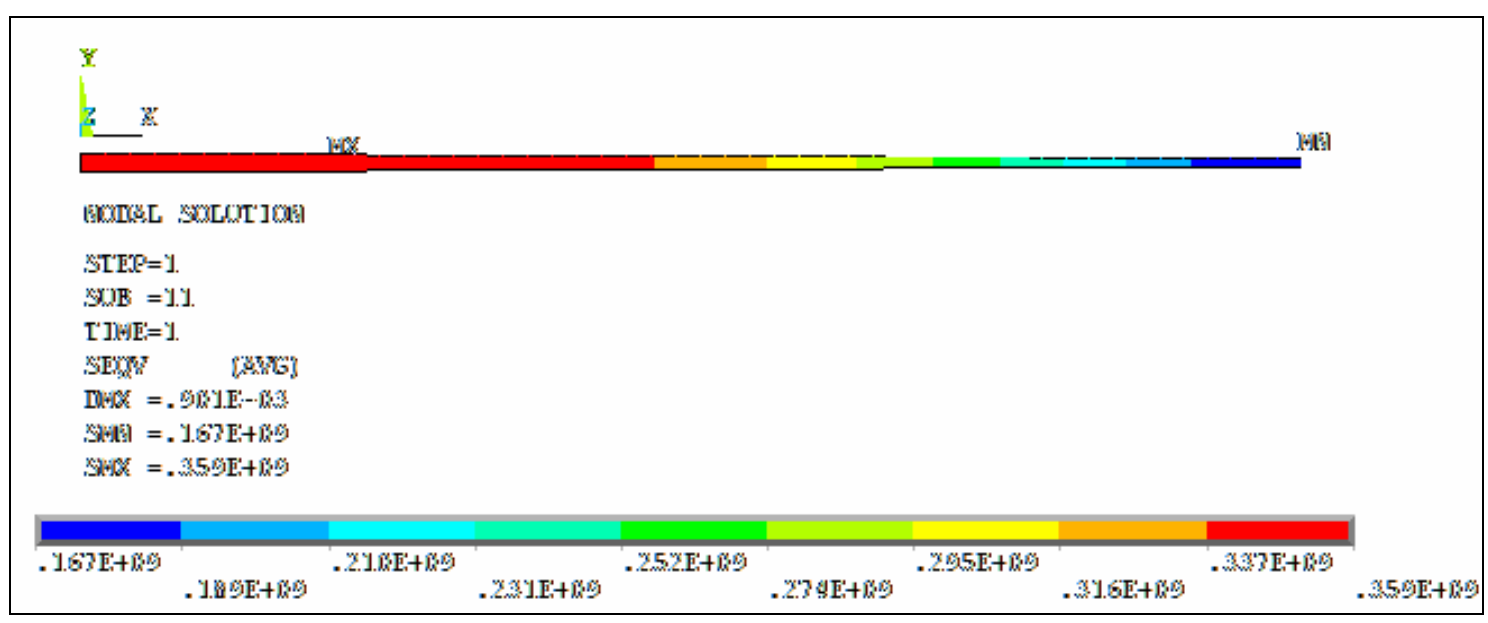

c) Plot of von-Mises stress at a fully plastic speed (at $\Omega_{2}=2.3506$ )

Figure 21. The finite element analysis of von-Mises stress fields at different rotational speeds. 


\section{References}

Laszlo F., 1948. Rotating disks in region of permanent deformation. NACA, pp. 1192.

Millenson M. B. and Manson S. S.,1948. Determination of Stresses in Gas-Turbine Disks Subjected to Plastic Flow and Creep. NACA, pp. 906.

Lee M. H. Wu, 1950. Analysis of plane-stress problems with axial symmetry in strain hardening range. NACA, pp. 2217.

Timoshenko S. P. and Goodier J. N.,1970. Theory of elasticity. McGraw-Hill, New York.

Calladine C. R., 1969. Engineering Plasticity. Pergamon Press, Oxford.

Srinath L., 2003. Advanced Mechanics of Solids II. Tata McGraw Hill, New Delhi.

Chakrabarty J., 1987. Theory of Plasticity. McGraw-Hill, New York.

Gamer U.,1984. Elastic-plastic deformation of the rotating solid disk. Ingenieur-Archiv. Springer, Vol. 54, pp. 345-354.

Güven U., 1992. Elastic-plastic stresses in a rotating annular disk of variable thickness and variable density. International Journal of Mechanical Sciences. Elsevier, Vol. 34, pp. 133-138.

Güven U., 1995. On the applicability of Tresca's yield condition to the linear hardening rotating solid disk of variable thickness. ZAMM- Journal of Applied Mathematics and Mechanics. Wiley, Vol. 75, pp. 397-398.

Eraslan A. N. and Orcan Y. 2002. Elastic-plastic deformation of a rotating solid disk of exponentially varying thickness. Mechanics of Materials. Elsevier, Vol. 34, pp. 423-432.

Eraslan A. N, 2003. Elasto-plastic deformation of rotating parabolic solid disks using Tresca's yield criteria. European Journal of Mechanics a Solids. Elsevier, Vol. 22, pp. 861-874.

Rees D. W. A., 1999. Elastic-plastic stresses in rotating disks by von-Mises and Tresca. ZAMM- Journal of Applied Mathematics and Mechanics. Wiley, Vol. 79, pp. 281-288.

You L. H. and Zhang J. J., 1999. Elastic-plastic stresses in a rotating solid disk. International Journal of Mechanical Sciences. Elsevier, Vol. 41, pp. 269-282.

You L. H., Tang Y. Y., Zhang J. J. and Zheng C. Y., 2000. Numerical analysis of elastic-plasticrotating disks with arbitrary variable thickness and density. International Journal of Solids and Structures. Elsevier, Vol. 37, pp. 7809-7820.

Eraslan A. N, 2002. Inelastic deformations of rotating variable thickness solid disks by Tresca and von-Mises criteria. International Journal of Computational Engineering Science. World Scientific Publishing Co., Vol. 3, pp. 89-101.

Alexandrova N. and Alexandrov S., 2004. Elastic-plastic stress distribution in a rotating annular disk. Mechanics Based Design of Structures and Machines. Taylor and Francis, Vol.32, pp. 1-15.

Alexandrova N., Alexandrov S., and Vila Real P. M. M., 2004. Displacement field and strain distribution in a rotating annular disk. Mechanics Based Design of Structures and Machines. Taylor and Francis, Vol.32, pp. 441-457.

Alexandrova N. and Vila Real P. M. M., 2007. Elastic-plastic stress distributions and limit angular velocities in rotating hyperbolic annular discs. Proceedings of the Institution of Mechanical Engineers, Part C: Journal of Mechanical Engineering Science. Professional Engineering Publishing, Vol.221, pp. 137-142.

Bhowmick S., Misra D. and Saha K. N., 2008. Approximate solution of limit angular speed for externally loaded solid rotating disk. International Journal of Mechanical Sciences. Elsevier, Vol.50, pp. 163-174.

Bhowmick S., Misra D. and Saha K. N., 2009. A parametric study on the growth of yield front in rotating annular disks. International Journal of Engineering, Science and Technology. Multicraft, Vol. 1, pp. 190-204.

\section{Biographical notes}

Shubhankar Bhowmick graduated in Mechanical Engineering from Pt. Ravi Shankar Shukla University, Raipur in 1999 and completed his ME in Mechanical Engineering (Machine Design) from Jadavpur University, Kolkata, in 2005. He is currently working as Assistant Professor, Department of Mechanical Engineering, National Institute of Technology, Raipur, Chattisgarh (India). He is engaged in teaching and research activities since the last 9 years. He was awarded with CSIR (SRF) Fellowship in the year 2007. His research area includes Stress and Deformation analysis, Investigation of static and dynamic behavioral analysis of axis-symmetric structures in elastic and post-elastic regime using variational principle. He is alsoactively engaged in FEA based industrial consultancy projects.

Dipten Misra is a Lecturer in the Department of Mechanical Engineering, Jadavpur University. His principal research area includes design of heat exchangers, heat transfer, finite element analysis and laser materials processing. Presently he is engaged in development of an experimental facility for validation of critical leak rate models and thermal fatigue monitoring system. He is also involved in simulation and experimental validation of laser cutting, welding, forming and laser cleaning of archaeological objects and items of cultural heritage. He has supervised a number of post graduate theses and research projects. He has several publications in international journals and conference proceedings. 
Kashi Nath Saha served Indian industry as design engineer in the sectors involving tea machineries, industrial fan and air handling equipment and other heavy machinery components for five years. He joined academia as lecturer in the Department of Mechanical Engineering, Jadavpur University in 1987. He is pursuing teaching and research in the area of machine elements design, stress, deformation analysis and design of structural elements and structural stability analysis. He has also carried out several experimental studies in the field of structural dynamics and supervised a number of post graduate thesis and research projects and has over 50 publications in international journals and conference proceedings.

Received January 2010

Accepted April 2010

Final acceptance in revised form May 2010 